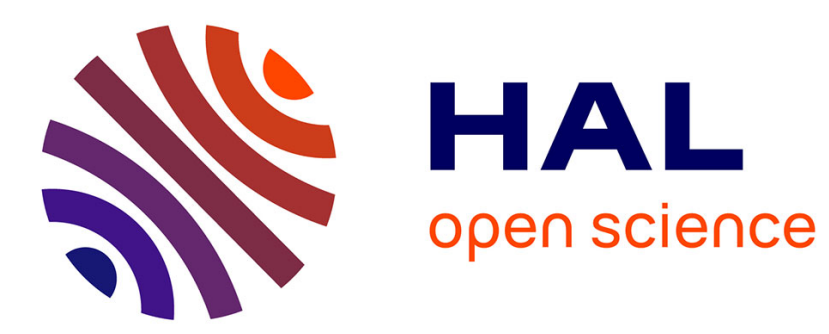

\title{
La constellation des quatre singes. Interprétation ethno-archéoastronomique des motifs de " El Carchi-Capuli " (Colombie, Equateur)
}

Dimitri Karadimas

\section{- To cite this version:}

Dimitri Karadimas. La constellation des quatre singes. Interprétation ethno-archéoastronomique des motifs de "El Carchi-Capuli " (Colombie, Equateur). Journal de la Société des américanistes, 1999, Volume 85, (Numéro 1), p. 115 -145. hal-01074632

\section{HAL Id: hal-01074632 \\ https://hal.science/hal-01074632}

Submitted on 15 Oct 2014

HAL is a multi-disciplinary open access archive for the deposit and dissemination of scientific research documents, whether they are published or not. The documents may come from teaching and research institutions in France or abroad, or from public or private research centers.
L'archive ouverte pluridisciplinaire HAL, est destinée au dépôt et à la diffusion de documents scientifiques de niveau recherche, publiés ou non, émanant des établissements d'enseignement et de recherche français ou étrangers, des laboratoires publics ou privés. 


\section{Persée}

http://www.persee.fr

\section{La constellation des quatre singes. Interprétation ethno-archéoastronomique des motifs de «El Carchi-Capuli » (Colombie, Equateur)}

\section{Dimitri Karadimas}

Journal de la société des américanistes, Année 1999, Volume 85, Numéro 1

p. $115-145$

\section{Voir l'article en ligne}

En partant d'une version d'un des mythes clés des Indiens miraña de l'Amazonie colombienne faisant référence à la constellation d'Orion, l'auteur se propose d'interpréter une série d'ornements corporels en or en provenance de la culture précolombienne du Nariño (Andes colombiennes) et de celle du Carchi (Andes équatoriennes). Le motif principal de ces pièces archéologiques est constitué par quatre singes disposés le plus souvent en trapèze de part et d'autre d'un personnage central. À cheval sur l'équateur céleste, la constellation d'Orion est liée aux deux astres majeurs : il semblerait que la disposition des singes et des personnages centraux sur les pièces archéologiques rende compte de cette particularité du ciel nocturne equatorial. Cette «structure orionis " pourrait également servir de scheme interprétatif à un ensemble iconographique commun aux sociétés andines lato sensu 1 .

\footnotetext{
Avertissement

L'éditeur du site «PERSEE » - le Ministère de la jeunesse, de l'éducation nationale et de la recherche, Direction de l'enseignement supérieur, Sous-direction des bibliothèques et de la documentation - détient la propriété intellectuelle et les droits d'exploitation. A ce titre il est titulaire des droits d'auteur et du droit sui generis du producteur de bases de données sur ce site conformément à la loi n $98-536$ du 1 er juillet 1998 relative aux bases de données.
}

Les oeuvres reproduites sur le site «PERSEE » sont protégées par les dispositions générales du Code de la propriété intellectuelle.

Droits et devoirs des utilisateurs

Pour un usage strictement privé, la simple reproduction du contenu de ce site est libre.

Pour un usage scientifique ou pédagogique, à des fins de recherches, d'enseignement ou de communication excluant toute exploitation commerciale, la reproduction et la communication au public du contenu de ce site sont autorisées, sous réserve que celles-ci servent d'illustration, ne soient pas substantielles et ne soient pas expressément limitées (plans ou photographies). La mention Le Ministère de la jeunesse, de l'éducation nationale et de la recherche, Direction de l'enseignement supérieur, Sous-direction des bibliothèques et de la documentation sur chaque reproduction tirée du site est obligatoire ainsi que le nom de la revue et- lorsqu'ils sont indiqués - le nom de l'auteur et la référence du document reproduit.

Toute autre reproduction ou communication au public, intégrale ou substantielle du contenu de ce site, par quelque procédé que ce soit, de l'éditeur original de l'oeuvre, de l'auteur et de ses ayants droit.

La reproduction et l'exploitation des photographies et des plans, y compris à des fins commerciales, doivent être autorisés par l'éditeur du site, Le Ministère de la jeunesse, de l'éducation nationale et de la recherche, Direction de l'enseignement supérieur, Sous-direction des bibliothèques et de la documentation (voir http://www.sup.adc.education.fr/bib/ ). La source et les crédits devront toujours être mentionnés. 


\title{
LA CONSTELLATION DES QUATRE SINGES. INTERPRÉTATION ETHNO-ARCHÉOASTRONOMIQUE DES MOTIFS DE « EL CARCHI-CAPULÍ » (COLOMBIE, ÉQUATEUR)
}

\author{
Dimitri KARADIMAS *
}

\begin{abstract}
En partant d'une version d'un des mythes clés des Indiens miraña de l'Amazonie colombienne faisant référence à la constellation d'Orion, l'auteur se propose d'interpréter une série d'ornements corporels en or en provenance de la culture précolombienne du Nariño (Andes colombiennes) et de celle du Carchi (Andes équatoriennes). Le motif principal de ces pièces archéologiques est constitué par quatre singes disposés le plus souvent en trapèze de part et d'autre d'un personnage central. À cheval sur l'équateur céleste, la constellation d'Orion est liée aux deux astres majeurs : il semblerait que la disposition des singes et des personnages centraux sur les pièces archéologiques rende compte de cette particularité du ciel nocturne équatorial. Cette "structure orionis " pourrait également servir de schème interprétatif à un ensemble iconographique commun aux sociétés andines lato sensu ${ }^{1}$.
\end{abstract}

MoTs CLÉs : mythologie, archéologie, ethnologie, astronomie, Miraña, Andes colombiennes et équatoriennes, Amazonie colombienne, orfèvrerie, iconographie précolombienne.

The Four Monkeys Constellation. Ethno-archaeoastronomical interpretation of the "El CarchiCapuli » motifs (Colombia, Equador)

Relying on a Miraña myth (Colombian Amazonia) about the constellation Orion, the author suggests an interpretation of some gold body ornaments from Nariño (Colombian Andes) and Carchi (Ecuadorian Andes) pre-Columbian cultures. The main motif of those archaeological objects is formed by four monkeys usually disposed in a trapeze around a central figure. On the celestial equator, the Orion constellation is linked to the sun and the moon; it seems that the monkeys and the central figure's position reflect this particularity of the nightly equatorial sky. This same "orionis structure" could also serve as an interpretative scheme for a set of iconographic motifs shared by Andean lato sensu societies.

KEY WORDS : mythology, archaeology, ethnology, astronomy, Miraña, Colombian and Ecuadorian Andes, Colombian Amazonia, gold ornaments, pre-Colombian iconography.

* Équipe de Recherche en Ethnologie Amérindienne (EREA) UPR 324 (CNRS), B.P. n 8, 7, rue Guy Môquet F-94801 Villejuif cedex et Centre d'Étude des langues Indigènes d'Amérique (CELIA) UMR 7595 (CNRS), 7, rue Guy Môquet F-94801 Villejuif cedex.

Journal de la Société des Américanistes 1999, 85 : p. 115 à 145. Copyright C Société des Américanistes. 
La constelación de los cuatro monos. Interpretación etno-arqueoastronómica de los motivos de "El Carchi-Capuli" (Colombia, Ecuador)

Partiendo de una versión de un mito clave de los indígenas mirañas de la Amazonía colombiana que se refiere a la constelación de Orion, el autor se propone interpretar un conjunto de ornamentos corporales de oro de las culturas precolombinas del Nariño (Andes colombianos) y del Carchí (Andes ecuatorianos). El motivo principal de estas piezas arqueológicas está constituido por cuatro monos generalmente repartidos simétricamente en trapezio alrededor de un personaje central. Sobre el ecuador celeste, la constelación de Orion está vinculada a los dos astros mayores ; parece que la disposición de los monos y de los personajes centrales de las piezas arqueológicas reproduce esta particularidad del cielo nocturno ecuatorial. Esta « structura orionis " podría igualmente servir de base interpretativa para una serie iconográfica común a las sociedades andinas lato sensu.

PALABRAS ClAVES : mitología, arqueología, etnología, astronomía, miraña, Andes colombianos y ecuatorianos, Amazonia colombiana, orfebrería, iconografia precolombina.

\section{INTRODUCTION}

Les pièces archéologiques en or originaires des Andes colombiennes ont ceci de passionnant que les interprétations qui existent à leur sujet sont inversement proportionnelles à leur nombre. Elles constituent, en quelque sorte, une terra non interpretata. Si la qualité et la quantité des matériaux archéologiques ne font pas défaut - bien qu'une majorité de pièces proviennent de fouilles sauvages, avec perte, donc, des contextes -, le contenu iconographique de ces figurines reste, lui, particulièrement résistant à toute tentative d'interprétation et ce, non sans raison. En effet, la plupart des cultures ayant produit ces artefacts ont disparu corps et âme avant la période coloniale et celles qui existaient encore au moment du Contact ont été dépouillées de leurs objets en or sans qu'aucune question quant aux motifs qu'ils étaient censés représenter n'ait été posée. En même temps qu'étaient fondus les ornements de nez, d'oreilles, les pectoraux, et autres diadèmes et ceintures en tumbaga pour récupérer les quelques grammes d'or mélangés au cuivre qu'ils contenaient, disparaissaient des motifs qui, aux yeux des populations amérindiennes, constituaient la raison d'être de ce qui était alors considéré par les conquérants comme de simples bijoux. Si les interprétations scolastiques ne sont pas légion en la matière, c'est qu'il n'existe jusqu'à présent aucune piste solide qui permettrait une interprétation systématique de ces motifs puisque les sociétés les ayant produits sont restées muettes à leurs propos.

Depuis la Conquête, les tombes ont livré leur contenu de parures en or et poteries richement peintes et ont pu élargir ainsi nos connaissances quant à l'utilisation post-mortem à laquelle étaient également destinés ces ornements. Cependant, ces sépultures sont restées tout aussi muettes pour ce qui est de l'iconographie représentée sur ces ornements; dans le meilleur des cas, il est possible de reconnaître une espèce animale ou végétale, ou encore des êtres hybrides combinant des formes humaines et des propriétés animales manifestées par certaines caractéristiques anatomiques patentes de l'espèce (la plus commune, sur ces représentations, étant les crocs de félins, signe de la nature prédatrice du personnage ou de la déité à qui ils sont prêtés).

Les pistes interprétatives proposées se tournent donc en majorité vers les populations indigènes actuelles pour interroger leurs mythologies et leur système de croyan- 
ces dans l'espoir de redonner un cadre symbolique et religieux aux représentations et figurines issues des fouilles de l'ensemble de ces civilisations sud-américaines ${ }^{2}$. Il va sans dire que cette approche est un pis-aller interprétatif dans la mesure où les populations actuelles ne sont pas nécessairement les héritières de ces cultures disparues et, même si tel était le cas, le système de croyance actuellement en vigueur est tributaire de plus de cinq siècles de changements et de bouleversements démographiques, économiques et sociologiques majeurs, ce qui laisserait à penser qu'un gouffre les sépare définitivement, rendant ainsi toute comparaison impossible. Nous pensons cependant que cette piste interprétative, fondée sur un comparatisme avec les systèmes de croyances des populations indigènes contemporaines, offre les meilleures possibilités pour la compréhension des anciennes sociétés des hautes terres colombiennes. Elle les place dans un continuum historique et sociologique - si chaotique qu'il puisse être - et présuppose le partage de certaines caractéristiques dans les modes de perception de l'environnement, dont les systèmes de croyances des cultures indigènes actuelles témoignent.

Le point de départ de cet article est un mythe recueilli en $1992 \mathrm{chez}$ les Miraña d'Amazonie colombienne et, à quelques variantes près, commun aux Bora, Miraña et Uitoto peuplant les berges du Caquetá et du Putumayo au sud de la Colombie. Les protagonistes du mythe, ainsi que son déroulement, présentent de fortes similitudes avec des personnages figurés sur une série d'ornements faciaux en provenance des cultures andines du Nariño et du Carchi-Cuasmal (période Capuli) d'époque préhispanique (étalées sur plus de deux mille ans - de 500 av. à 1500 apr. J.-C. - et réparties de nos jours entre la Colombie et l'Équateur), cultures dont les zones d'extension couvrent justement les sources du Putumayo et, dans une moindre mesure, du Caquetá.

Il devient ainsi possible, à l'aide du mythe miraña et de la disposition particulière des personnages sur les pièces archéologiques, d'apporter un éclairage novateur sur cette série de pièces ornementales en or et d'en fournir une interprétation en termes de représentation astrale. En regardant ces pièces archéologiques avec des «yeux miraña ", celles-ci devenaient des illustrations surgies du passé d'un mythe entendu tout juste il y a quelques années. La disposition des personnages présents sur ces pièces d'orfèvrerie, comme celle des personnages du mythe miraña, évoquerait la constellation d'Orion qui, comme nous le montrerons, occupe une place particulière et centrale dans le ciel étoilé circuméquatorien. Après avoir dégagé la structure principale de ces ornements corporels, elle pourra être appliquée à des séries de pièces d'orfèvrerie - mais aussi à des motifs de poteries - provenant d'autres cultures des Andes colombiennes et équatoriennes possédant une même disposition générale. La constellation des «Quatre Singes " nous renvoie à une "structure Orionis " qui pourrait permettre d'unifier des représentations hétérogènes et hétéromorphes présentes sur un ensemble jusqu'ici disparate de pièces archéologiques.

Lorsque les motifs de ces représentations liées à la constellation d'Orion sont associés à des personnages importants, nous pensons qu'aux yeux des sociétés en question, la motivation première de leur utilisation semble être une figuration d'un destin post-mortem sous forme d'étoile ; tout comme il semble y avoir une identification - de son vivant - de celui qui les porte avec l'astre solaire ou lunaire (l'un et l'autre étant liés à ce groupement d'étoile, respectivement à l'est et à l'ouest, mais nous $\mathrm{y}$ reviendrons). 


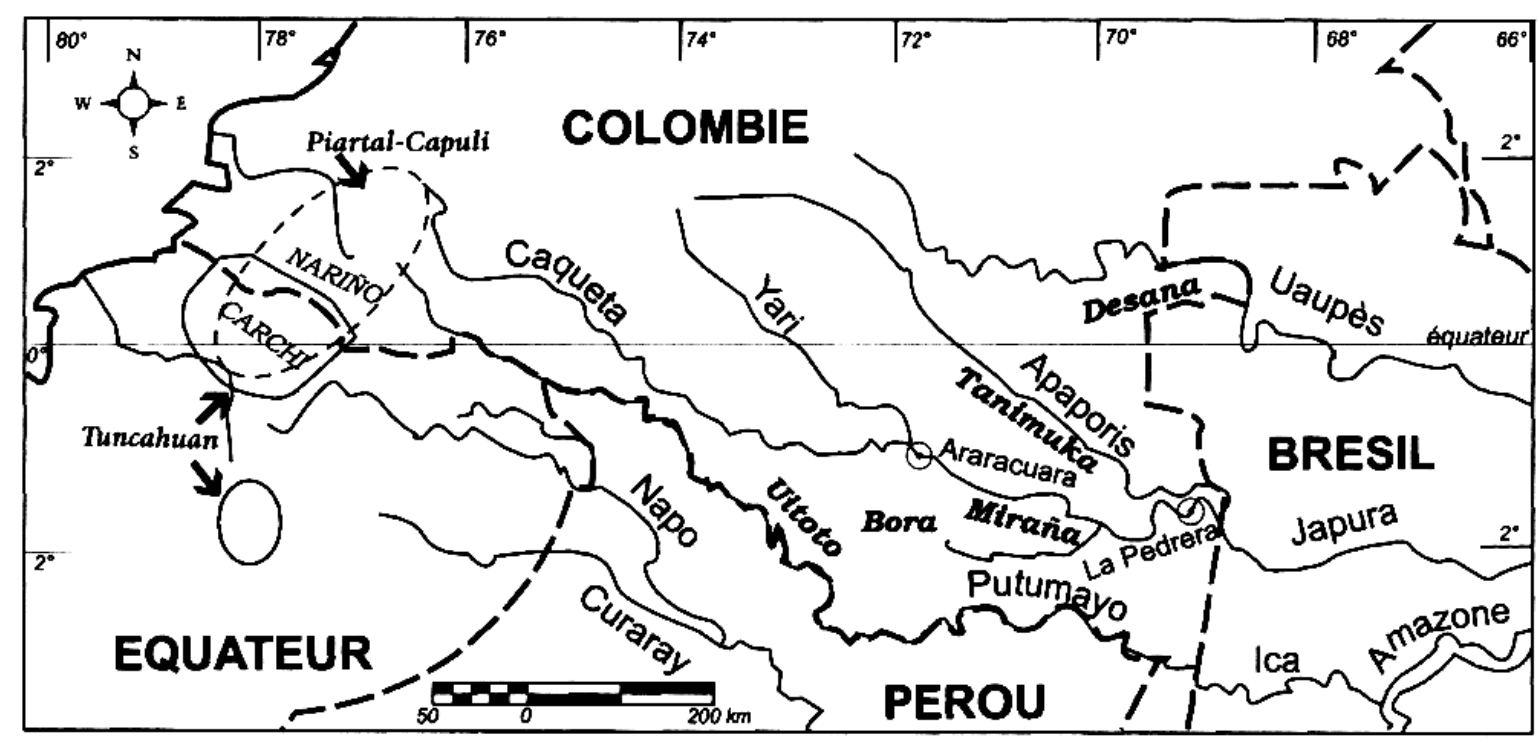

CARTE 1. - Répartition des groupes et des aires archéologiques.

\section{PrÉSENTATION DE LA ZONE}

Il nous faut présenter rapidement les deux pôles de la zone concernée par notre étude. D'un côté, le Moyen-Caquetá / Putumayo, milieu tropical humide des basses terres et, de l'autre, les hautes terres du Carchí en Équateur et du Nariño en Colombie.

Les Miraña occupent en habitat dispersé une large portion du Caquetá entre les chutes d'Araracuara et les rapides de La Pedrera avec une plus forte densité autour de l'embouchure du Cahuinari, alors que les Uitoto sont établis sur les affluents de la rive gauche du Putumayo en descendant celui-ci (Igara-paraná et Cara-paraná). Il existe également des communautés Bora et Uitoto au Pérou depuis leur déportation lors de la période du boom du caoutchouc au début de ce siècle. Que ce soit en Colombie ou au Pérou, ces deux populations vivent actuellement dans des maisons sur pilotis construites autour d'une maloca, anciennement maison plurifamiliale, faisant office de lieu cérémoniel en plus d'abriter le chef de maisonnée et sa famille, qui représente aussi chaque communauté locale. Les sociétés qui habitent cet espace peuvent être considérées comme vivant principalement de la chasse, de la pêche, de la culture du manioc (amer et doux), ainsi que d'autres cultigènes (plantain, ananas, arachides, patates douces, etc.) parmi lesquels les fruits du palmier parépou (Bactris gasipaes, désigné comme chontaduro en Colombie) occupent une place rituelle de premier ordre. Toutes les sociétés du Caquetá / Putumayo utilisent la coca et le tabac qu'elles considèrent comme des plantes sacrées.

Les chutes d'Araracuara - lieu de première importance mythique pour les populations actuelles du Caquetá - constituent également, pour les basses terres, un site archéologique de premier ordre dont l'occupation remonte " avant l'ère chrétienne pour la tradition « camani " qui est remplacée par celle dite " nofurei » vers 800 apr. J.-C. " (Andrade, $1986: 8$ ) ; et, plus précisément, " 100 av. J.-C. " (ibid. : 16). Le site archéologique de La Pedrera regroupe une quantité importante de pétroglyphes liés à ceux d'Araracuara. 
La majorité des pièces archéologiques que nous nous proposons d'interpréter dans un premier temps ne proviennent pas des basses terres mais d'un ensemble régional couvrant le nord de l'Équateur et le sud de la Colombie et remontant à deux périodes : une première qui va de 500 av. à 500 apr. J.-C., et qui concerne deux pièces en provenance de la culture « Tuncahuán », et une seconde qui va de 500 à 1500 apr. J.-C. et qui concerne le style Carchi-Cuasmal (période Capulí; Lavallée \& Lumbreras, 1985) ainsi que l'art Piartal pour le reste des pièces. La période des styles CarchiCuasmal et Piartal a été considérée, du point de vue stylistique et de la technique dite en « négatif " des décors de poteries - sur laquelle nous reviendrons - comme une continuation de la première (ibid. : 245).

Ces cultures sont situées en majorité dans la zone des hauts plateaux, avec quelques extensions sub-andines. Étrangement, les animaux et végétaux représentés sur les pièces archéologiques - comme les singes par exemple - appartiennent plus à la faune et à la flore des basses terres qu'à celles des Andes. ${ }^{3}$

De plus, et pour souligner l'étroite relation entre le moyen Caquetá-Putumayo et les sociétés des hauts plateaux andins situés près des sources de ces fleuves, une série de figurines en terres cuites en provenance de Carchi-Cuasmal représentent un personnage assis sur un tabouret, chiquant de la coca et portant toutes un bonnet identique (des « coqueros », ibid. : 246). Or, d'après M. Guyot - ethnographe des Bora-Miraña dans les années soixante-dix - un bonnet était également, jusque dans ces mêmes années, un des insignes bora caractérisant la génération des aînés (1974, fig. 7), alors que les tabourets personnalisés sont, jusqu'à présent, des marques d'un statut privilégié (chef de maloca) pour les Miraña et les Bora. Il faut souligner qu'à notre connaissance, aucune autre société de la région n'associe traditionnellement un bonnet et un tabouret comme insignes des personnages de première importance au sein du groupe, ce qui laisserait supposer une relation plus étroite entre ces deux ensembles culturels.

Les liens commerciaux qui ont existé entre les sociétés des basses terres du Caquetá-Putumayo et celles des hautes terres colombiennes et équatoriennes sont largement connus, et remontent à une période préhispanique bien qu'ils n'aient peut-être pas atteint le moyen Caquetá (Uribe, 1986). Parmi les objets et les biens échangés apparaissent des singes, des aras et des perroquets (dont les petits sont élevés et apprivoisés par les femmes des sociétés des basses terres) que les populations des hautes terres gardaient comme animaux domestiques à la mode amazonienne. La cire d'abeille en provenance des basses terres est également très appréciée dans la mesure où elle sert au modelage des motifs des céramiques polychromes " en négatifs " ${ }^{4}$, tout comme dans les moulages en or à cire perdue. Or c'est justement sur ce type de céramiques largement répandu dans la culture Tuncahuán qu'apparaissent les figurations les plus anciennes de singes ou du groupement des Quatre Singes ( $c f$. infra).

Parmi les objets échangés faisant le trajet inverse, c'est-à-dire des Andes vers les basses terres, semblent se trouver des ornements corporels en or. Les premières informations concernant le fleuve Japurá (Caquetá), sur lequel, d'après les sources historiques, les Miraña semblent avoir toujours vécu, remontent au XVII ${ }^{\mathrm{e}}$ siècle lorsque, lors de l'expédition de Pedro Teixeira en 1638, Mauricio de Heriarte identifie le Japurá avec le Rio de Ouro. L'ethnohistorien brésilien Antonio Porro fait remarquer à 
propos de ce Rio de Ouro qui préoccupa les colons à la recherche du territoire de l'Eldorado :

"O fabuloso « Rio do Ouro ", de que Pedro Teixeira teve notícia ao subir o Solimões e que seria o pomo de disputa de fronteiras com Espanha, recebeu esse nome porque os índios da foz do Japurá usavam pequenos ornamentos de ouro que les vinham do norte, do alto rio Negro e do Uaupés, que os índios chamavam Iquiari. Acuña preocupo-se em localizar a região aurifera, mas conseguiu somente definir o trecho inferior da rota e identificar os seus agentes : "A quatorze léguas dessa aldeia, que chamamos de Ouro, da banda do Norte, sai a boca do rio Yupurá, que é por onde se entra no Ouro [...] onde do pé de uma serra que elí está o tiram os naturais em grande quantidade ; e este ouro é todo em pontas e grãos de bom tamanho dos quais formam, à força de batê-los, as placas que, já disse, perduram nas orelhas e narizes ».(Acuña $1941: 240$, 237-8, in Porro 1985 : 9-10).

Après l'examen d'autres sources historiques, le même auteur conclut à propos des régions aurifères d'où provenaient ces objets en or rencontrés chez les populations peuplant l'embouchure du Japurá : "Isto sugere que a região aurifera onde os Manaus se abasteciam não estaria no alto rio Negro, mas muito mais a oeste, no sopé dos Andes colombianos, onde nasce o Uaupés. " (ibid.)

Nous pouvons donc admettre que, pour le moins vers la fin de la période précolombienne, les régions du Japurá et du Vaupés étaient en contact avec les sociétés dites Cacicares des Andes colombiennes d'où provenaient, grâce à des routes d'échange pour un commerce intertribal, des ornements corporels en or ; comme le laisserait penser la localisation des sources de ces deux fleuves. Les populations amazoniennes portaient ces ornements en or dans les oreilles et le nez selon la même mode qui prévalait alors dans les Andes.

Il existe deux hypothèses en ce qui concerne le schéma général du peuplement de la zone. Selon Lathrap (1970), les cultures de forêts auraient émigré de l'embouchure de l'Amazone en remontant le fleuve à la recherche des terres alluviales particulièrement fertiles (varzeas). Ces cultures auraient alors colonisé les Andes par l'intermédiaire des affluents de l'Amazone (dont le Caquetá, fleuve à "eaux blanches ", d'origine andine). Le schéma de colonisation serait donc, suivant cette hypothèse, celui d'une " remontée vers les Andes " et non d'une « descente vers les basses terres " accompagnée d'un appauvrissement culturel, présentant les sociétés des basses terres comme des systèmes culturels « déchus » (seconde hypothèse, défendue par Meggers, 1971). De plus, l'archéologie de la zone andine de Pasto-Nariño fait apparaître qu'il n'existe pas de traces de peuplement avant la période d'intégration (500 apr. J.-C.), ce qui laisserait penser que la colonisation de l'aire est postérieure à la celle des basses terres avoisinantes.

Quoi qu'il en soit de ces deux hypothèses, il faut souligner que les échanges n'ont pas été à sens unique mais que les rapports entretenus entre les zones indiquent au contraire d'intenses emprunts mutuels (voir en particulier les travaux de Ramírez de Jara, 1996 a, b ; Renard-Casevitz et al., 1988 ; Cárdenas-Arroyo \& Bray, 1998). Il nous paraît ainsi essentiel de noter l'existence de relations étroites entre les sociétés des hautes et des basses terres; elles se laisseraient appréhender, en plus des échanges commerciaux, par exemple par une même attention portée sur des phénomènes astraux. Le lien entre ces deux ensembles serait illustré, dans le cas spécifique qui nous intéresse, par une perception et une représentation similaires de la constellation 
d'Orion. En d'autres termes, nous retiendrons bien plus l'option d'intenses contacts et d'échanges continus que celle d'une diffusion de traits culturels d'un ensemble à un autre, même si, comme nous allons le montrer, ces ensembles semblent partager une cosmogonie et un système de représentation communs concernant le ciel étoilé.

\section{PRÉSENTATION DES PIÈCES ARCHÉOLOGIQUES}

Avant de nous atteler à la description des céramiques en provenance de l'art Tuncahuán, il nous faut décrire les ornements faciaux en or qui constituent l'essentiel des figurations des quatre singes remontant à la période Capulí de l'art Carchi / Nariño.

Il s'agit le plus souvent de paires d'ornements d'oreilles constituées sur un même modèle général. Deux singes sont placés à chaque extrémité d'un arc de cercle convexe alors que la figure centrale, placée, elle, en dessous de cet arc de cercle dans la partie concave, varie d'une paire à l'autre dans la forme. Cette figure centrale représente, soit des motifs géométriques variés ( $1 \mathrm{~b}, \mathrm{c}, \mathrm{e})$, soit un personnage accroupi qui semble suspendu par la tête (1f), soit ce même personnage avec une tête apparaissant en relief alors que le reste du corps est plat ( $\mathrm{lg}$ ), soit une représentation astrale avec des rayons apparents - tous deux en relief - (1d), soit, encore, des motifs phytomorphes et, plus précisément, un racème de fruits suspendu qui semble correspondre aux fruits du palmier parépou (Bactris gasipaes) ${ }^{5}$ et qui apparaît lui aussi en relief (1h).

Certains de ces ornements d'oreilles ne comportent pas deux mais quatre singes ( $1 \mathrm{~b}, 1 \mathrm{i})$, et les ornements de nez $(1 \mathrm{a}, 1 \mathrm{j})$ dépourvus de figure centrale sont également constitués de quatre singes qui, lorsqu'ils étaient portés, entouraient la bouche. C'est en nous appuyant sur ces ornements de nez que nous pensons que les ornements d'oreilles doivent être analysés comme ayant un modèle de base correspondant à quatre singes (deux fois deux, une paire de singes sous chaque oreille), et une partie centrale, qui est répétée sur chacune des boucles. Le modèle général de ces pièces est donc celui de quatre singes situés symétriquement, par paire, de part et d'autre d'une figure centrale ou, en son absence - le cas des ornements de nez - symétriquement par rapport au visage, ou à la tête du porteur, qui devient alors cette figure centrale absente.

Les éléments constants de ces ornements d'oreilles et de nez sont les singes qui se retrouvent sur pratiquement toutes les pièces en provenance de cette culture ${ }^{6}$, alors que la figure centrale prend des formes variées et interchangeables. Par une méthode de commutation, les personnages accroupis, les motifs géométriques, les figurations astrales et le racème de fruits sont équivalents les uns aux autres. Ils ne représentent, suivant l'hypothèse que nous comptons accréditer grâce au mythe miraña, que des états de transformations d'un même personnage qui devient alternativement chacune de ces figurations. En bref, nous pensons que ces différentes représentations centrales peuvent être interprétées dans le sens d'une série de transformations que subit un seul et même personnage mythique, et qu'elles constituent donc un ensemble homogène.

Il reste un dernier point de description des pièces qui semble négligeable au premier abord mais qui revêt, en fait, une importance primordiale dans l'interprétation générale que nous en proposons. Lorsque les singes apparaissent sur les ornements de nez $(1 \mathrm{a}, \mathrm{lj})$ ou sur les boucles d'oreilles par groupe de quatre $(\mathrm{lb}, 1 \mathrm{i})$, leur disposition 


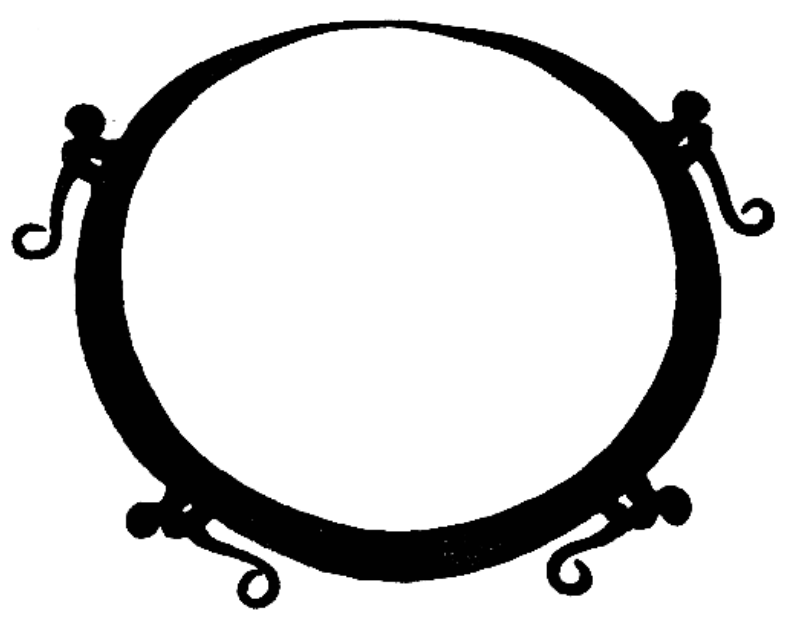

FIG. 1a - Ornement de nez Capuli ; 4 singes.
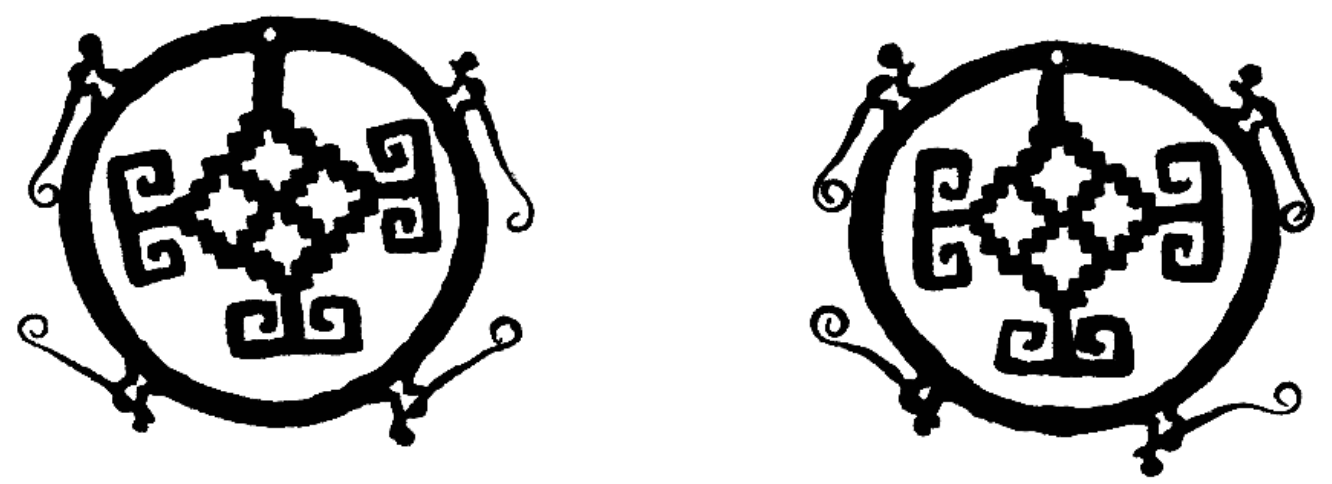

FIG. 1b. - Ornements d'oreilles Capuli : 2 x 4 singes, motif central géométrique (Astre ?) MO25.405-06.
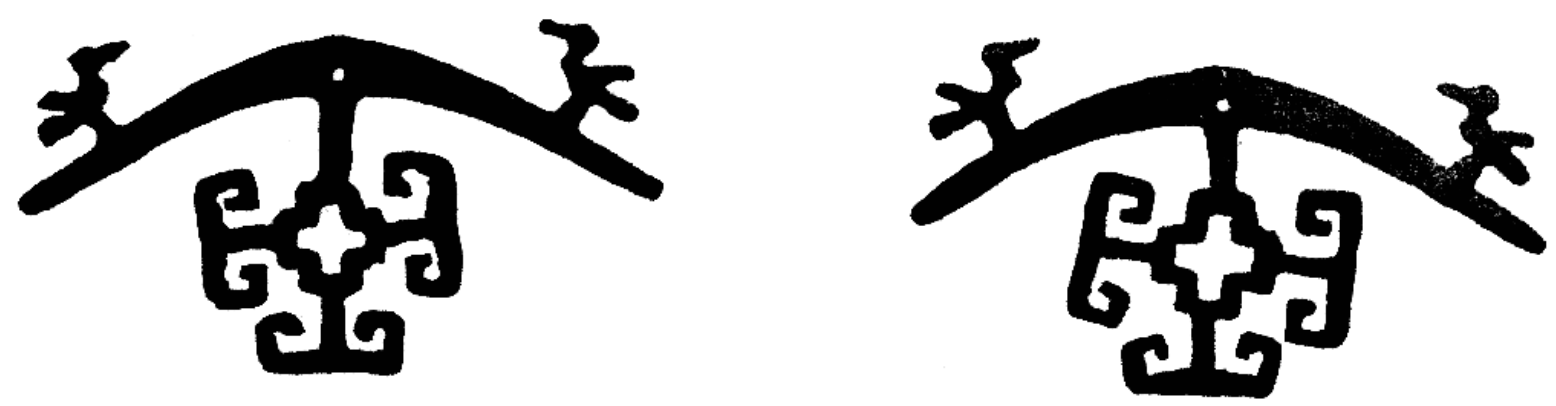

FIG. 1c. - Ornements d'oreilles Capuli : 2 x 2 volatiles, motif central géométrique (Astre ?) MO25.408-09. 

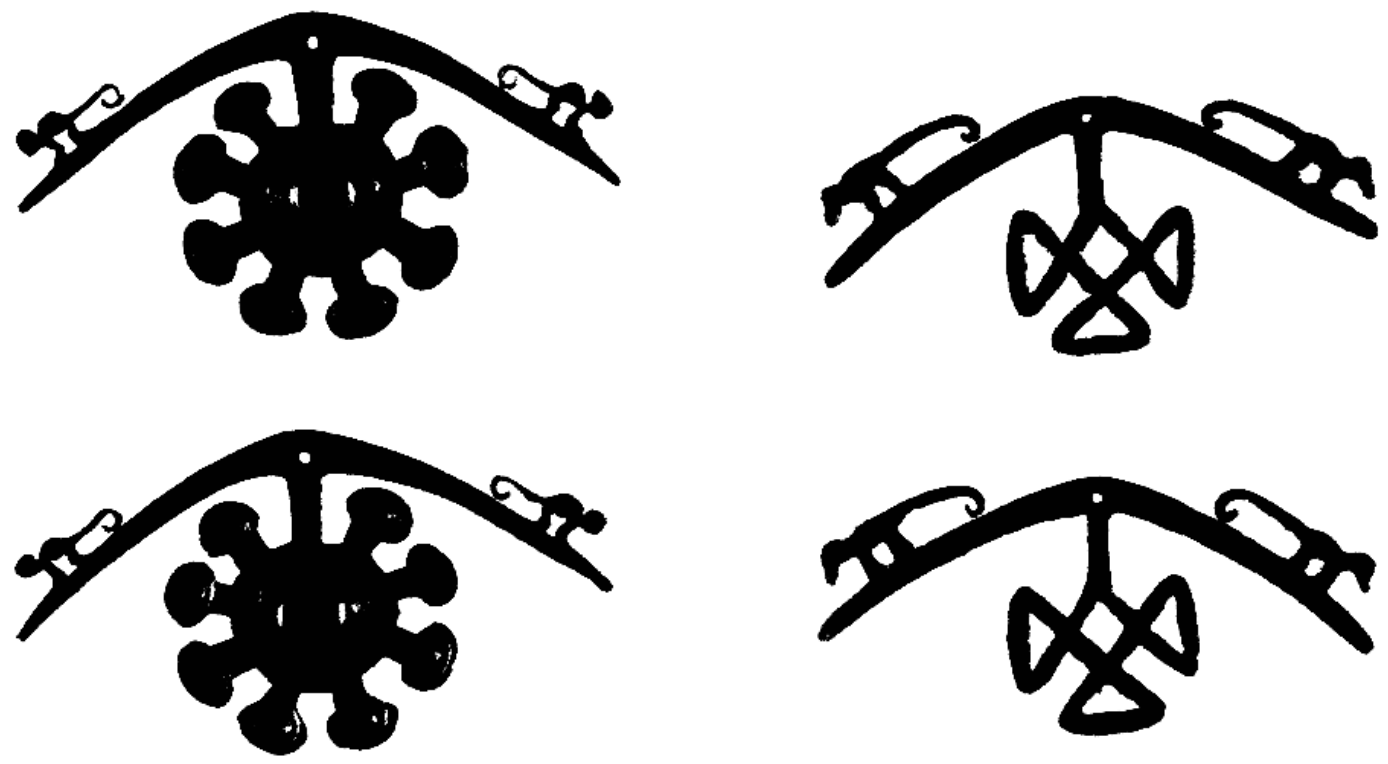

FIG. 1d. - Ornements d'oreilles Capuli, 2 x 2 singes avec motif central en forme d'astre.

FIG. 1c. - Ornements d'oreilles Capuli 2 x 2 kinkajous ; motif central géométrique (Astre?).
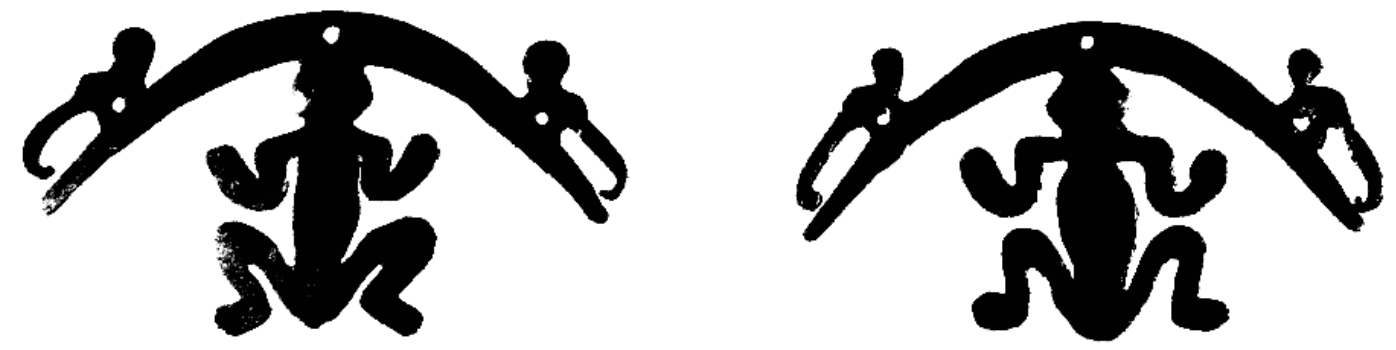

FIG. If. Ornements d'oreilles Capuli : $2 \times 2$ singes avec personnage central accroupi, bras relevés.
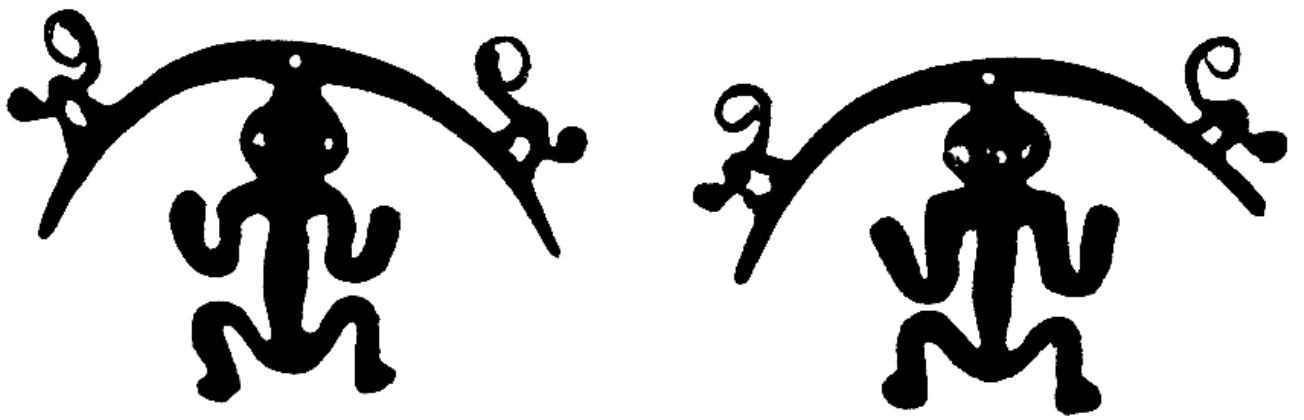

FIG. Ig. - Ornements d'oreilles Capuli : $2 \times 2$ singes avec personnage central accroupi, tête enflée (Astre ?). 

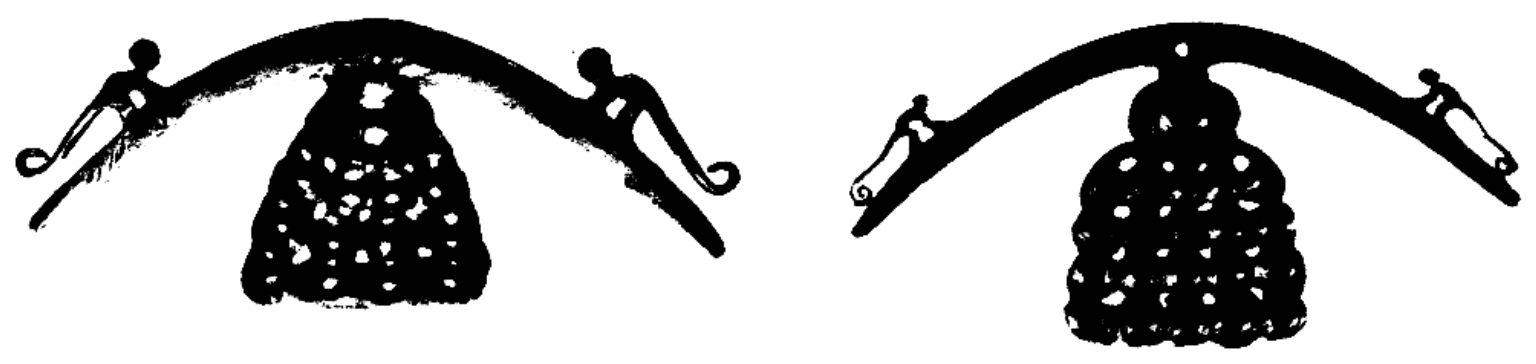

FIG. 1h. - Ornements d'oreilles Capulí : 2 × 2 singes avec racème de palmier en relief et ajouré (chacun des spécimens provient d'une paire différente).
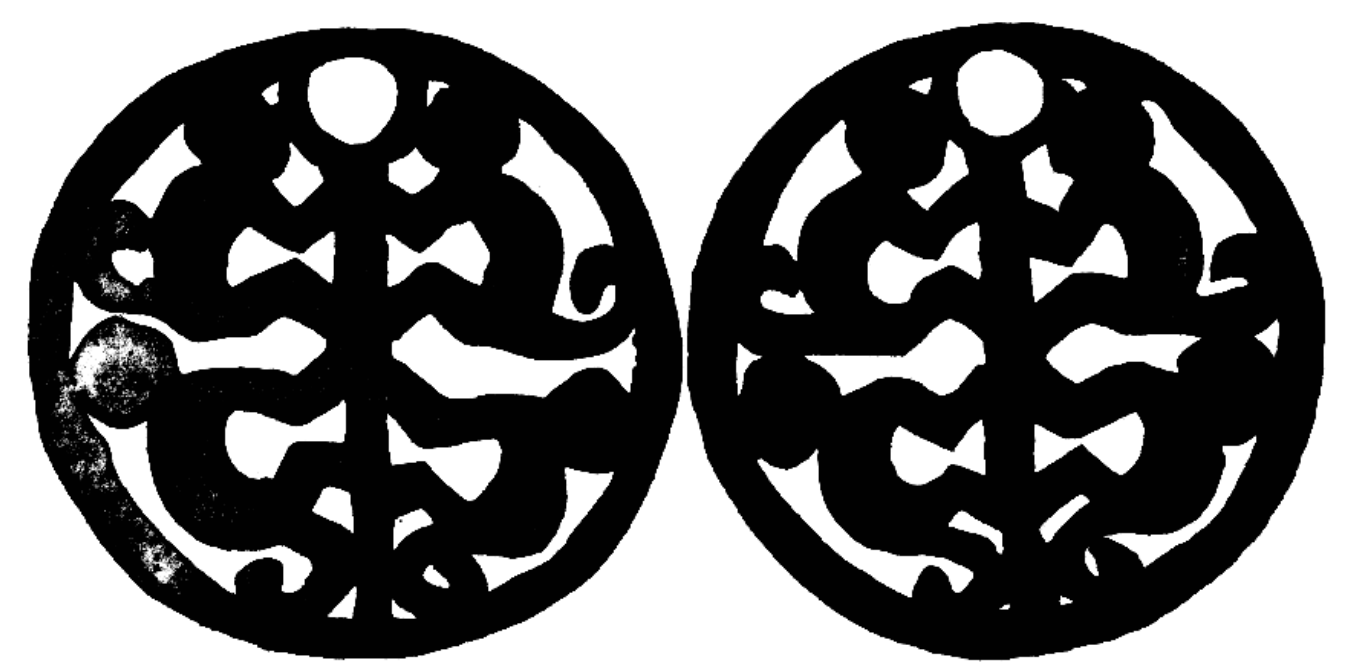

FIG. 1i. - Ornement d'oreilles Capulí : $2 \times 4$ singes répartis symétriquement autour d'un axe vertical, les quatre têtes forment un trapèze sur chaque ornement (pièces MO 31.638-39).

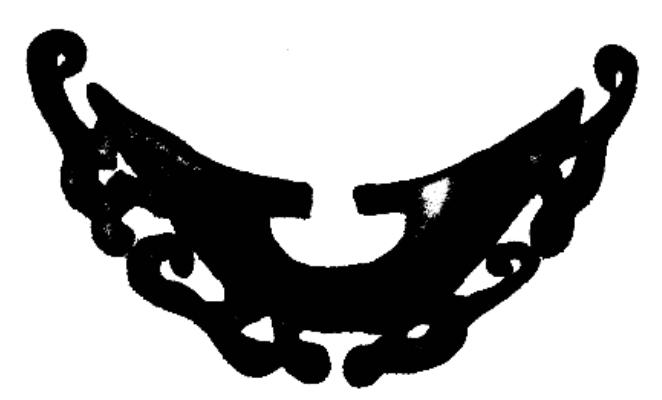

FIG. 1j - Ornement de nez Capuli constitué de quatre singes en position renversée (MO 20.971). 
sur le cercle, ou à l'intérieur de celui-ci, n'est pas équidistante, mais l'écartement des deux singes du haut est toujours différent de celui de la paire du bas. Les quatre singes ne forment pas les angles d'un vague carré, même imparfait, mais un trapèze dont le côté bas est toujours différent, en taille, de celui du haut (dans la mesure où il existe un "bas " et un " haut " puisque ces ornements d'oreilles sont suspendus par un trou présent sur les pièces d'orfèvreries). Nous insistons sur cette particularité de la disposition des singes puisqu'elle va réapparaître, comme constante, sur des pièces archéologiques en provenance d'autres cultures précolombiennes et parce qu'elle reproduit fidèlement la disposition des quatre étoiles majeures de la constellation d'Orion ( $c f$. infra). La paire d'ornements d'oreilles (1i) possède très distinctement cette disposition : les têtes des quatre singes sont disposées symétriquement en trapèze suivant un axe diamétral vertical.

Il est possible d'ajouter à cet ensemble de pièces issues de la phase Capulí de l'art Nariño-Carchi, des figurations de singes présentes sur des poteries de la période Tuncahuán qui sont, elles, beaucoup plus anciennes (époque du développement régional ; env. 500 av.-500 apr. J.-C.). Nous reproduisons ici le motif interne de deux coupes.
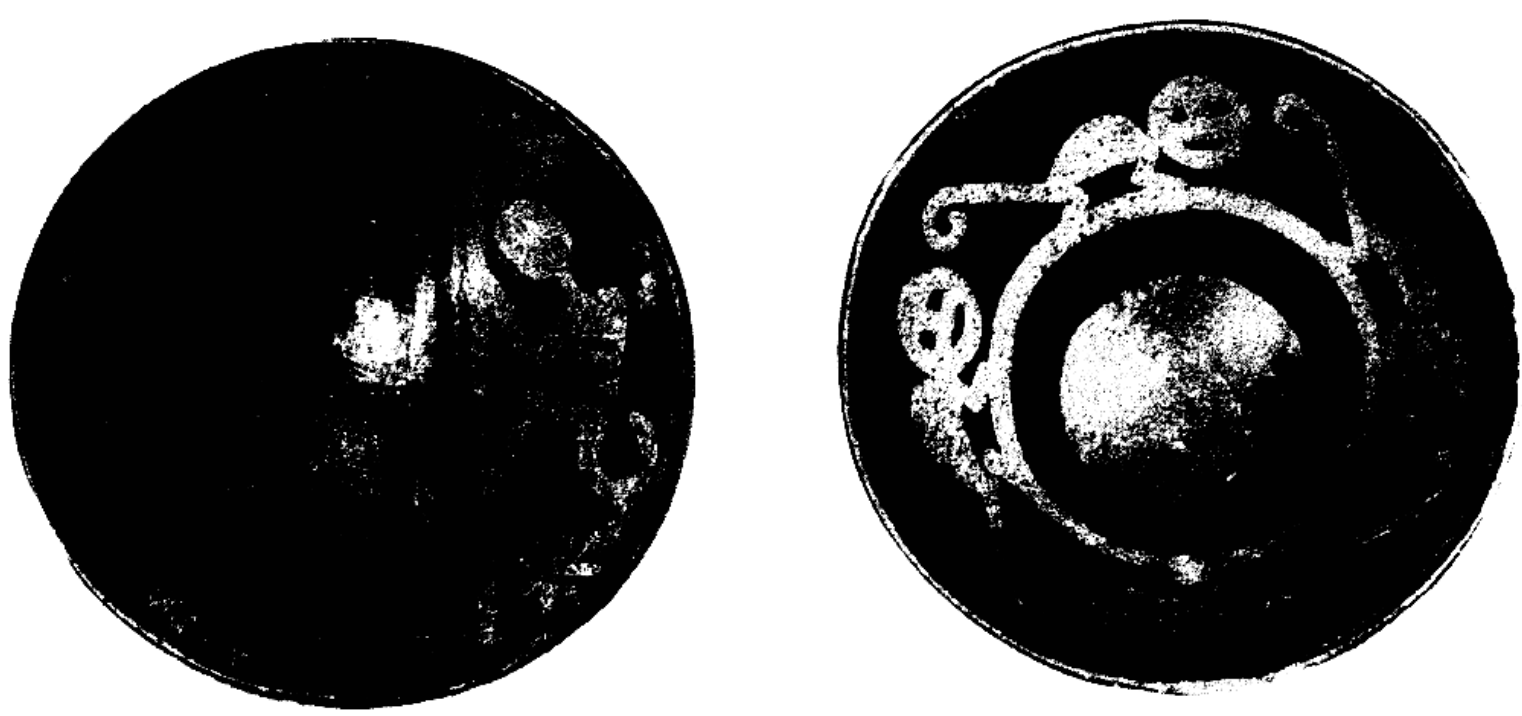

FIG. 2a \& 2b. - Intérieurs de coupes Tuncahuán.

L'intérieur de la première représente des silhouettes de singes se faisant face tête-bêche et entourés de quatre motifs que nous pensons être des étoiles (suivant la manière la plus simple - pour nos sociétés — de dessiner une étoile c'est-à-dire par une double croix, tracée, comme l'ensemble des motifs; en négatif sur fond noir). L'intérieur de la coupe est divisé suivant une symétrie bilatérale ; les singes et les étoiles sont répartis suivant cet axe de symétrie.

L'intérieur de la seconde coupe représente directement quatre singes : corps en profil et têtes de face, disposés sur un cercle au centre duquel un disque apparaît en négatif.

Ces intérieurs de coupes rappellent le décor de céramiques du Carchi-Nariño (période Piartal, $\mathrm{IX}^{\mathrm{e}}$ au XVI $\mathrm{XI}^{\mathrm{e}}$ siècle ap. J.-C.) qui utilisent également le motif des quatre 
singes placés symétriquement de part et d'autre d'une ligne médiane, accompagné, le plus souvent, par quatre petits cercles centraux (Figures $3 \mathrm{a} \& 3 \mathrm{~b}$ ) :
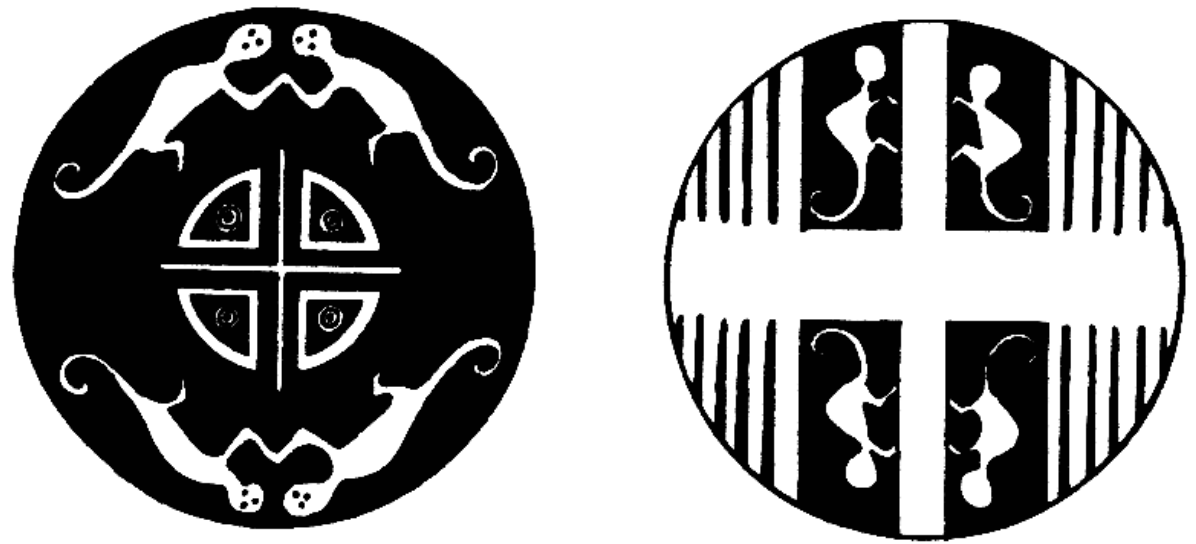

FIG. 3a \& 3b. - Intérieurs de coupes Piartal (4 singes).

Enfin, l'intérieur d'une dernière coupe (Figure 4) représente deux singes placés tête-bêche l'un en face de l'autre. Cette représentation est intéressante à plus d'un titre puisqu'elle semble combiner plusieurs conceptions en une seule. En effet, il est possible de reconnaître que les membres antérieurs et postérieurs des deux singes forment également ceux d'une chauve-souris en position de vol, dont l'intérieur des ailes est occupé par les corps des singes (il suffit de rétablir les singes dans une position de symétrie diamétrale et non plus axiale pour que les ailes de la chauve-souris soient déployées dans le même sens). Cette chauve-souris est dépourvue de tête dans la mesure où elle doit être reconnaissable dans les deux positions (elle n'a, en quelque sorte, ni haut ni bas). L'intérieur de la coupe n'est pas entièrement noirci ; ne sont laissées en noir que les parties devant représenter les ailes de la chauve-souris. Au centre de son corps est représenté un cercle blanc. Des deux extrémités de son corps sortent deux bandes blanches remplies de rectangles noirs s'évasant vers les bords du contenant. Il ne nous est pas encore possible de démontrer que la chauve-souris aux ailes déployées est une représentation du ciel nocturne dans lequel s'inscrivent les « singes » qui représentent des constellations; c'est pourtant dans ce sens que va notre interprétation.

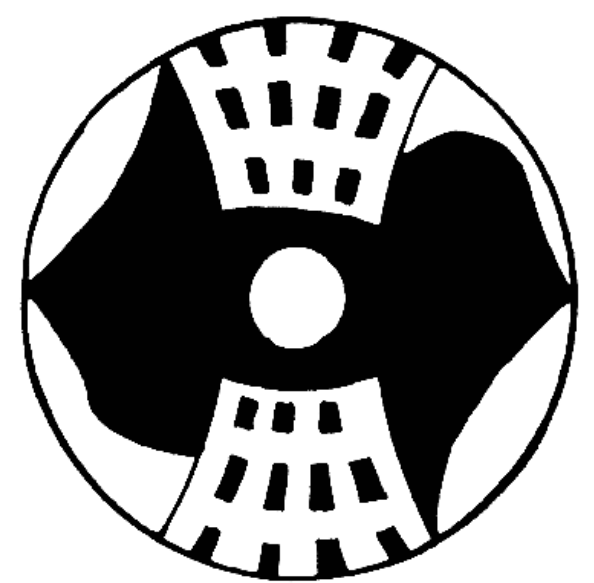

Fig. 4. - Intérieur de coupe Piartal. 2 singes dans les ailes d'une chauve-souris. 
Nous nous arrêterons dans un premier temps à cette série de pièces pour en proposer une interprétation en fonction d'une lecture miraña rendue possible à l'aide du mythe du "Souffleur de Sarbacane ", héros culturel et personnage central d'une longue épopée que nous résumerons au maximum pour n'en retirer que les éléments qui nous intéressent au premier chef ${ }^{7}$.

\section{LE MYTHE MIRAÑA : UNE INTERPRÉTATION ASTRONOMIQUE}

" Astre " ( ( du jour " pour le soleil, et « de la nuit » pour la lune), suite à son mariage avec une femme dénommée Kinkajou (Poto flavus) se trouve confronté à ses beaux-frères douroucoulis (Aotus sp. $)^{8}$. Après les avoir poursuivis toute la nuit dans les arbres grâce aux reflets de leurs yeux, Astre (" de la nuit ») les voit disparaitre au petit matin dans un " trou d'ara " (nid d'ara) en haut d'un tronc mort de palmier. Il grimpe au tronc et penche la tête dans le trou pour apercevoir ses beaux-frères, mais ceux-ci, de l'intérieur du trou d'ara, agrippent sa tête, la tirent, et le décapitent. La tête d'Astre tombe au fond du palmier creux dans le monde souterrain des poissons avec lequel communique le palmier du "trou d'ara " et se transforme en racème de parépou (Bactris gasipaes).

"Souffleur de Sarbacane", fils d'Astre, grandit orphelin de père. Adolescent, il apprend par la bouche de chauve-souris que ses oncles maternels, les douroucoulis, ont tué son père et vivent cachés en parasites en haut des quatre poteaux centraux de la maloca en se faisant nourrir par sa mère. Pour venger son père, il décide de les tuer, les fait cuire, les mange et replace leurs crânes en haut des quatre poteaux centraux. La mère se fait également tuer puis dévorer par son fils en se faisant passer pour un kinkajou qui arrive au petit matin pour voler les fruits du verger de la maloca. Après une longue épopée, le mythe se termine alors que le héros part chez les poissons pour récupérer " la chair de son père ", un noyau de parépou qu'il rapporte sur terre, morceau de l'astre diurne avec lequel ce palmier est aujourd'hui identifié.

Cette version du mythe appelle plusieurs commentaires. En premier lieu, les Miraña ne font pas de distinction nominale entre l'astre diurne et nocturne ; l'un étant au jour ce que l'autre est à la nuit et parce que le héros culturel "Souffleur de Sarbacane " a repris le nom de son père "Astre " en même temps qu'il rapporte le noyau de parépou sur terre. C'est le contexte mythique qui rend le plus souvent compte de l'identité solaire ou lunaire d'Astre. Dans le mythe, c'est l'entité Lune qui poursuit ses beaux-frères douroucouli. La décapitation du personnage a donc lieu à l'aube, juste avant que le soleil ne se lève à l'est; elle a été précédée par la fuite des singes nocturnes par le "trou d'ara " (comportement que partage cette espèce avec le kinkajou puisque, fuyant la lumière du jour, ils passent la journée à dormir en se servant des nids d'ara et autres cavités d'arbre comme gîte). Or ce « trou d'ara » qui se trouve en haut d'un palmier dans le mythe, est également le toponyme, en miraña, des chutes de Araracuara. "Araracuara " (littéralement " trou d'ara ") n'est d'ailleurs que la traduction en lingua geral (tupi) de cette toponymie que partagent plusieurs groupes de la région.

En un sens, ce que le mythe présente comme une décapitation du personnage « Astre » dans le trou d'ara du palmier par lequel ont fui les douroucoulis n'est que la retranscription dans un langage mythologique d'un phénomène astronomique associé à une particularité de la topographie locale. En effet, l'escarpement d'Araracuara laisse passer le Caquetá dans une large faille que son cours a creusé durant des siècles, 
laissant apparaître, de chaque côté, de grandes falaises et formant un long couloir tumultueux mais rectiligne. Le lieu est investi par les aras qui viennent y nicher en grand nombre. Enfin, les chutes sont situées exactement à l'ouest du territoire miraña à $0^{\circ} 35^{\prime}$ sud par rapport à l'équateur et $72^{\circ} 23^{\prime}$ de longitude ouest. Il existe ainsi une rencontre de ce lieu avec le trajet équinoxial du soleil ainsi qu'avec l'équateur céleste et, ce d'autant plus que le lieu surplombe l'ensemble du territoire miraña ; c'est derrière cette barrière rocheuse que disparaissent quotidiennement de la vue des habitants du moyen Caquetá les astres nocturnes et l'astre diurne. Il est donc possible de conclure que la poursuite des douroucoulis par Lune se faisait d'est en ouest (si l'on prend en compte les emplacements relatifs du territoire miraña et des chutes de Araracuara).

Dans le mythe miraña, l'astre nocturne se fait décapiter par des singes qui l'ont précédé dans ce " trou d'ara ». Suivant une compréhension astronomique du mythe, il devrait s'agir de corps célestes situés autour de ce lieu, précédant dans le courant de la nuit le trajet de la lune. La seule constellation qui remplit ces caractéristiques est celle d'Orion, laquelle - lorsqu'elle est associée à la lune - est appelée « constellation des douroucoulis » en miraña. Les singes nocturnes sont des étoiles d'Orion. L'association entre les singes nocturnes, les étoiles et la lune est forte chez les Bora (même langue et système culturel que les Miraña) puisque, d'après une information recueillie par J. Gasché et al., les Bora associent les douroucoulis aux étoiles ; ils prétendent, en effet, qu'en les visant en pleine nuit, ils doivent faire attention à ne pas confondre les étoiles avec leur proie à cause de l'éclat des yeux des primates qui brillent dans la nuit comme des étoiles. L'association avec la lune proviendrait du fait que les douroucoulis ont, selon les Bora, une activité accrue les nuits de lune (1996:220). Un séjour récent (janvier 1999) chez les Miraña du moyen Caquetá m'a permis de confirmer cette déduction ; Orion est bien désigné comme la constellation des douroucoulis. Enfin, la mère du héros qui, dans le mythe, prend l'apparence d'un kinkajou, est explicitement reliée à Vénus par les Miraña.

Mettons momentanément entre parenthèses - dans le déroulement du mythe la transformation de la tête d'Astre en racème de parépou pour passer directement à la vengeance du fils d'Astre qui tue ses oncles maternels et place leurs crânes en haut des quatre poteaux centraux de la maloca.

L'architecture des malocas miraña respecte une orientation est-ouest matérialisée par la poutre faîtière aussi désignée comme le « chemin du soleil ». Le toit à double pente est supporté par quatre piliers centraux dont l'apex vient se placer aux trois quarts des arbalétriers pour former un carré. Vu de haut, la poutre faîtière répartit ces quatre poteaux en deux paires symétriques : deux au nord et deux au sud puisque la faîtière suit un axe est-ouest. Or l'acte que réalise le héros culturel de déposer les quatre crânes des douroucoulis en haut de ces poteaux équivaut, pour les Miraña, à en faire des représentations d'étoiles dans la mesure où la maloca est un modèle réduit du monde dans lequel le toit occupe la place du ciel. Pour les Miraña, société anciennement anthropophage, les crânes des ennemis suspendus dans le toit de la maloca sont la manifestation des âmes des morts qui partent dans le ciel pour briller éternellement sous forme d'étoiles ( $c f$. Karadimas, 1997). Ce sort n'est réservé qu'aux guerriers morts au combat, ou rituellement sous les dents de leurs ennemis, ainsi qu'aux chefs de maloca - représentants du soleil sur terre -, enterrés dans leur maloca au centre de ces quatre poteaux centraux ( $c f$. Guyot, $1976: 163$ ). Les singes nocturnes sont ainsi au 
nombre de quatre, comme les poteaux centraux de la maloca miraña, et les crânes placés en haut dans le toit selon la disposition de ceux-ci reproduisent la distribution des quatre étoiles d'Orion dans le ciel étoilé. Cette inscription architecturale de la constellation n'est d'ailleurs pas un fait isolé dans la région. Les Tanimuka, voisins septentrionaux des Miraña, possèdent une architecture et une symbolique de leur maloca similaire à celle des Miraña ; pour M. von Hildebrand, les quatre poteaux centraux de la maloca tanimuka sont $\alpha, \beta, \gamma$ et $\chi$ d'Orion $(1987: 242)$.

Les quatre singes nocturnes du mythe miraña représenteraient donc les quatre étoiles d'Orion, identifiés par von Hildebrand comme Bételgeuse $(\alpha)$, Rigel ( $\beta$ ), Bellatrix $(\gamma)$ et Saiph $(\chi)$ d'Orion. Ces quatre étoiles forment un trapèze dans le ciel nocturne qu'il serait donc possible d'associer au trapèze que forment les singes figurés sur les pendentifs en or de la période Capulí de l'art du Carchi-Nariño puisque, contrairement aux bâtisseurs de maloca, les orfèvres de ces sociétés n'avaient pas de contraintes architecturales les obligeant à figurer Orion par un carré.

Il faut souligner que, dans son ouvrage sur la faune précolombienne du Nariño, E. Rodríguez, estime que certaines des espèces de singes représentées sur l'orfèvrerie de cette région sont également des douroucoulis ou « singes nocturnes » 9 .

Son interprétation varie par contre pour les autres espèces simiesques représentées sur les pièces en or ; toutes ne représenteraient pas des douroucoulis, et certaines espèces comme Ateles $s p$. seraient également représentées. Nous pensons cependant qu'il s'agit là d'une variation sur le thème des douroucoulis, dans la mesure où la disposition des singes respecte celle des étoiles d'Orion.

Un argument supplémentaire pour associer les représentations de singes de la région du Nariño avec des étoiles est que les groupes indigènes de la période historique semblent faire ce lien. Ainsi, dans un extrait proposé par S. Elías Ortiz à propos de l'importance des singes dans la cosmogonie des groupes du Nariño, celui-ci relate une discussion avec un missionnaire espagnol : "Le prêtre espagnol P. Bazares nous raconta que, lors d'une de ses incursions dans son vaste domaine de prêche du Tablón à la recherche de plantes médicinales, il rencontra une famille indigène du groupe des Aponte dans une cabane si éloignée de tout commerce humain que les idées religieuses y étaient complètement défigurées. Pour gagner la confiance de cette famille, le Père Bazares parla longuement du paradis et de ses joies, mais il se rendit compte que l'évocation de ces délices ne faisait aucune impression sur ses auditeurs. Le chef de la famille le tira de son étonnement : y a-t-il ou non des singes dans le ciel ? " (S. Elías Ortiz, $1934: 315$, in : E. E. Rodríguez B., 1992: 80, notre traduction). Cette question posée par l'indigène au prêtre peut être interprétée différemment si l'on prête à l'indigène une croyance en un destin post-mortem de la gent simiesque ou non. Il apparaît cependant plus probable que cette interrogation fait référence à l'identification mythologique de certaines étoiles avec des singes à l'instar du mythe miraña, puisque la question de l'indigène se réfère au ciel et non spécifiquement à un paradis. En conséquence de quoi il nous faudrait analyser l'ensemble des représentations de singes sur les poteries et l'orfèvrerie de cette région comme étant des références faites à des héros mythiques devenus, par une série de transformations, des constellations et ce d'autant plus que ces représentations en suivent le tracé.

L'identification, par les Miraña, du kinkajou avec Vénus, qui est donc la « sœur » classificatoire des quatre douroucoulis (Orion) peut également être associée à l'art du 
Nariño. En effet, toujours selon E. E. Rodríguez, Poto flavus est représenté dans $93 \%$ des collections en provenance du Nariño, suivi de près par des représentations de singes $(80 \%)$ et de chauve-souris ( $73 \%)(1992: 99)$. Cette association majeure entre kinkajou et singes dans les représentations de l'art précolombien de cette région doit être prise en compte comme un argument en faveur de l'identification du procyonidé avec Vénus. Des pendentifs d'oreille de la même période (Figure 5a \& 5b) (MO 25.366-67, MO 25.407) associent distinctement des kinkajous - reconnaissables à leur museau proéminent - avec des représentations d'astres que l'on pourrait facilement interpréter comme le soleil (une sphère centrale en relief entourée de rayons, terminés chacun par une autre forme globuleuse) si la cosmogonie miraña n'incitait à privilégier l'identification avec l'étoile du matin.
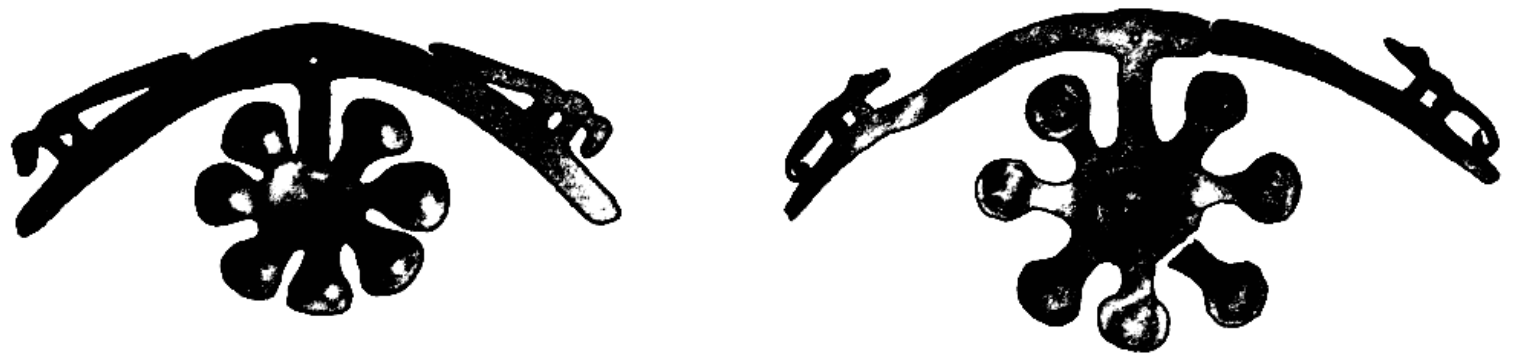

FIG. 5a \& 5b. — Ornement d'oreilles Capuli : kinkajous et astre (Vénus) (MO 25.366 \& 25.407).

Un point reste cependant obscur dans les représentations que donnent les anciennes sociétés du Nariño de la constellation d'Orion. En effet, cette constellation n'est pas seulement formée par quatre étoiles en trapèze, mais également par trois étoiles alignées en son centre et connues comme le "Baudrier d'Orion » ou les " Trois Rois " $(\delta, \varepsilon$ et $\zeta$ d'Orion). Or pratiquement toutes les pièces que nous avons étudiées jusqu'à présent en sont exemptes. Toutefois, d'autres ornements d'oreilles provenant de la même période présentent un motif géométrique central dans un style identique aux ornements d'oreille précédemment illustrés ( $\mathrm{lb}$ ), mais ne possèdent que trois singes (Figure 6).

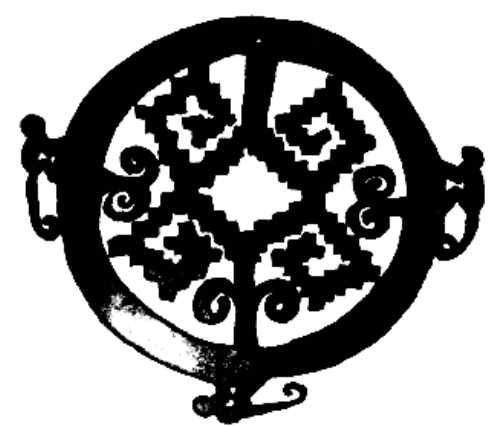

FIG. 6. - Ornement d'oreille Capuli, (3 singes). MO 25.204.

Il n'est pas possible d'affirmer avec certitude que ces successions de trois singes correspondent aux trois étoiles centrales de la constellation, dans la mesure où ils ne respectent ni l'angle formé entre ces étoiles, ni leur alignement. En revanche, une 
poterie de la période Piartal (Figure 7a) semble parfaitement respecter cet alignement, associant deux singes tête-bêche et une suite de trois étoiles entre ces derniers.

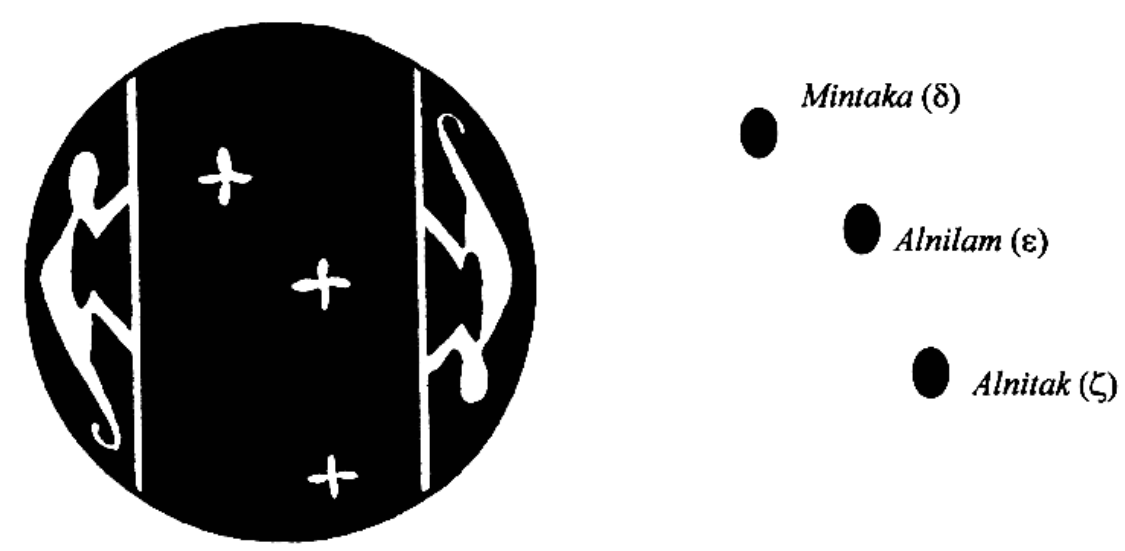

FIG. 7a. - Intérieur de poterie Piartal : 2 singes têtes-bêches, trois étoiles centrales FIG. 7b. : - Le Baudrier d'Orion.

Si nous superposons à présent le motif de la poterie en provenance de la période Tuncahuan (Figure 2a) et le motif de la poterie Piartal (Figure 7a), la constellation d'Orion apparaît dans sa totalité (Figure 7c).

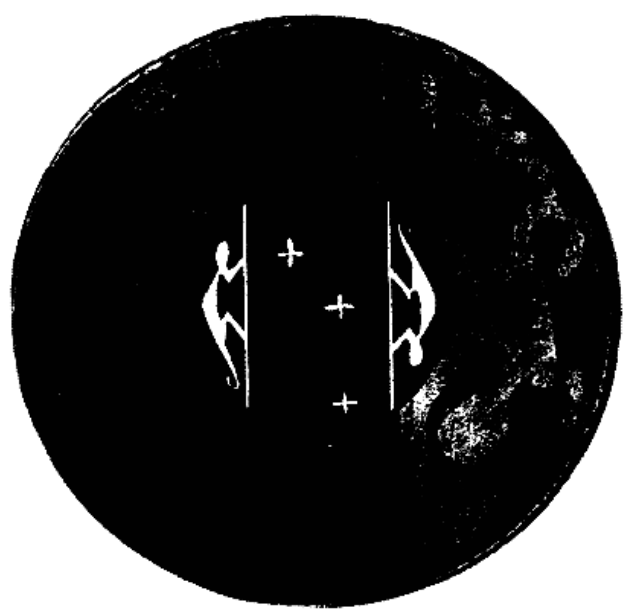

FIG. 7c. - Superposition des motifs de 2a \& 7a : la constellation d'Orion vue dans son ensemble.

Cette superposition des motifs des deux poteries est, bien sûr, artificielle. Il n'est cependant pas interdit d'effectuer cette manipulation puisque la scène représentée dans l'intérieur des poteries est parfaitement identique sur les deux contenants aux groupements d'étoiles près et qui se complètent ${ }^{10}$. On retiendra malgré tout qu'il n'existe pas — dans l'état actuel de l'inventaire des pièces archéologiques - de représentation combinée des deux groupements d'étoiles formant la constellation aux yeux des sociétés du Carchi-Nariño ${ }^{11}$. Il semble ainsi plus probable que ces sociétés associent d'un côté, le trapèze de la constellation et, de l'autre, les trois étoiles centrales (Mintaka, Alnilam et Alnitak). 
Il parait maintenant possible, par extrapolation, de considérer les singes des ornements corporels arrivant par groupe de trois comme étant ces trois étoiles centrales du Baudrier d'Orion.

Mais retournons au mythe miraña pour expliquer les représentations de la figure centrale présente sur les ornements corporels. Il resterait en effet à associer la tête décapitée d'Astre avec sa matérialisation transformée qui est le racème de bactris, pour que l'ensemble des pièces archéologiques que nous nous sommes proposés d'interpréter (pendentifs d'oreilles et de nez du Nariño) prenne un sens tout à fait similaire à celui du mythe miraña.

En effet, les fruits du parépou sont considérés par les Miraña comme des fruits solaires à cause de leur couleur rouge, mais aussi du fait que leur chair, extrêmement grasse et difficile à digérer, donne souvent, lorsqu'elle est consommée en grande quantité, de sérieuses coliques attribuées à son origine solaire ${ }^{12}$. Pour les Miraña toujours, chaque fruit de Bactris gasipaes est en fait un morceau de soleil, et le noyau, à l'origine, une hypostase de la cervelle transformée du personnage mythique. La totalité du racème de parépou, composé d'une multitude de fruits, représente la tête d'Astre - chairs et cervelle - dans laquelle les chairs de la tête se sont transformées en celles des fruits et chaque partie de la cervelle dans les différents noyaux qui composent le racème ${ }^{13}$.

Ainsi, toutes les représentations situées sur les ornements en or en dessous de l'arc de cercle convexe, en commençant par celles qui indiquent clairement l'identité solaire de sa représentation (1d) doivent être associées à la transformation successive de la tête du personnage principal (1f) dont le traitement en relief $(1 \mathrm{~g})$ semble souligner l'attention qui a voulu être donnée à cette partie du corps de l'entité astrale ${ }^{14}$ (en dissociant toutefois ces représentations astrales de celles liées aux kinkajous qui, par hypothèse, se réfèrent à Vénus, $c f$. figures $5 \mathrm{a} \& 5 \mathrm{~b}$ ). On peut en effet souligner que toutes les parties des ornements en or qui sont rehaussées par leur mise en relief — soleil, tête du personnage et racème - sont également des parties qui subissent une transformation dans le mythe miraña.

Pour autant, nous ne faisons que postuler cette identité du personnage avec l'astre solaire puisqu'il n'est pas encore possible de démontrer que le personnage de ces ornements en or est à associer à l'astre du jour ou de la nuit, c'est-à-dire qu'il s'agisse, comme dans le mythe, de figurations de transformations de la tête d'Astre. Il n'y a que les singes y figurant qui doivent être considérés comme représentant la constellation d'Orion puisqu'ils partagent la même disposition trapézoïdale avec celle-ci, tout comme leur ancrage de part et d'autre de l'axe équinoxial (matérialisé par le soleil sur la pièce $1 \mathrm{~d})$.

Il nous manquerait, pour cela, un élément majeur, à savoir la figuration de la scène de décapitation par quatre personnages - si possible de forme simiesque - qui rendrait ainsi valide notre démonstration. Or, dans l'état actuel de nos connaissances, cette figuration est absente des pièces archéologiques en provenance de Carchi-Nariño et de la période Capulí. On la retrouve en revanche sur des séries de pectoraux en provenance de diverses cultures précolombiennes des Andes colombiennes, mais relativement distantes de la zone du Carchi-Nariño. Le recours à ces pectoraux pour les besoins de notre démonstration pose toutefois plusieurs problèmes. En effet, nous nous autoriserions à comparer des pièces archéologiques qui sont issues - aussi bien 
dans le temps que dans l'espace - de cultures hétérogènes à celle productrice des pièces ornementales examinées jusqu'ici mais avec laquelle, de par la proximité géographique et les contacts attestés, la culture miraña aurait pu avoir eu des échanges, rendant compte des congruences entre le mythe et les pièces ornementales.

En définitive, si l'hypothèse que nous formulons est valide, à savoir que l'ensemble des pièces archéologiques examinées fait référence à la constellation d'Orion, il faudrait que cette constellation possède des caractéristiques si particulières qu'elle ait été perçue et retranscrite de façon similaire dans les différentes sociétés précolombiennes, alors même que ces représentations sont matérialisées par des acteurs hétéromorphes sur les pièces d'orfèvrerie. En d'autres termes, nous postulons l'existence d'une structure d'Orion liée au soleil, à la lune, et à leur course ; structure qui est exploitée par ces sociétés sur leur iconographie pour l'associer au personnage qui portait ces ornements et réaliser ainsi sur celui-ci ce que la constellation accomplie avec l'astre diurne et nocturne.

Il nous importe donc maintenant de décrire l'éphéméride d'Orion autour de l'équateur avant de pouvoir passer à l'examen d'autres pièces archéologiques; le détour par l'astronomie est destiné surtout à voir comment se comporte le cycle d'Orion.

UN PEU D'ASTRONOMIE

\section{Configuration générale du ciel nocturne autour de l'équateur}

La première particularité du ciel nocturne pour un observateur situé aux alentours de la ligne imaginaire de l'équateur est la bipartition parfaite du ciel étoilé selon un axe est-ouest, alors que les points cardinaux nord et sud présentent des points fixes autour desquels se " déroule ", pendant les douze heures de nuit, le ciel étoilé. Cette bipartition suit la courbe équinoxiale du soleil, qui se matérialise par l'absence d'ombre portée lorsque le soleil est au zénith (ces deux périodes équinoxiales ont lieu à la troisième semaine de mars et à la même semaine en septembre en prenant comme point de référence l'équateur), ainsi que par un parcours du soleil ayant lieu au centre des deux points extrêmes que sont les trajets solsticiaux ( 21 juin et 22 décembre). L'année se trouve ainsi découpée suivant quatre périodes de trois mois suivant les allers et retours du soleil selon trois points placés sur l'horizon oriental et trois autres, leur répondant de façon symétrique, sur l'horizon occidental (voir Zuidema, 1989 [1980] ; Magaña, 1988 : 98 et passim).

Les astronomies équatoriennes semblent ainsi axées sur la ligne d'horizon (ascension et déclinaison verticale des constellations, formant, dans leur déplacement, un angle droit avec la ligne d'horizon). Ces systèmes astronomiques prennent plus particulièrement en compte les accidents topographiques figurant sur la ligne d'horizon (des "marqueurs d'horizon ») lorsqu'ils sont associés à une constellation précise ${ }^{15}$, permettant de les caractériser comme des " astronomies de l'horizon " (voir Aveni \& Urton, 1982 ; Magaña, 1988). À l'Est, les étoiles donnent l'impression de " sortir " (verticalement) de sous la terre, à l'Ouest elles semblent " repartir " ou " tomber " sous celle-ci ; leur configuration est alors renversée par rapport à l'Est. 


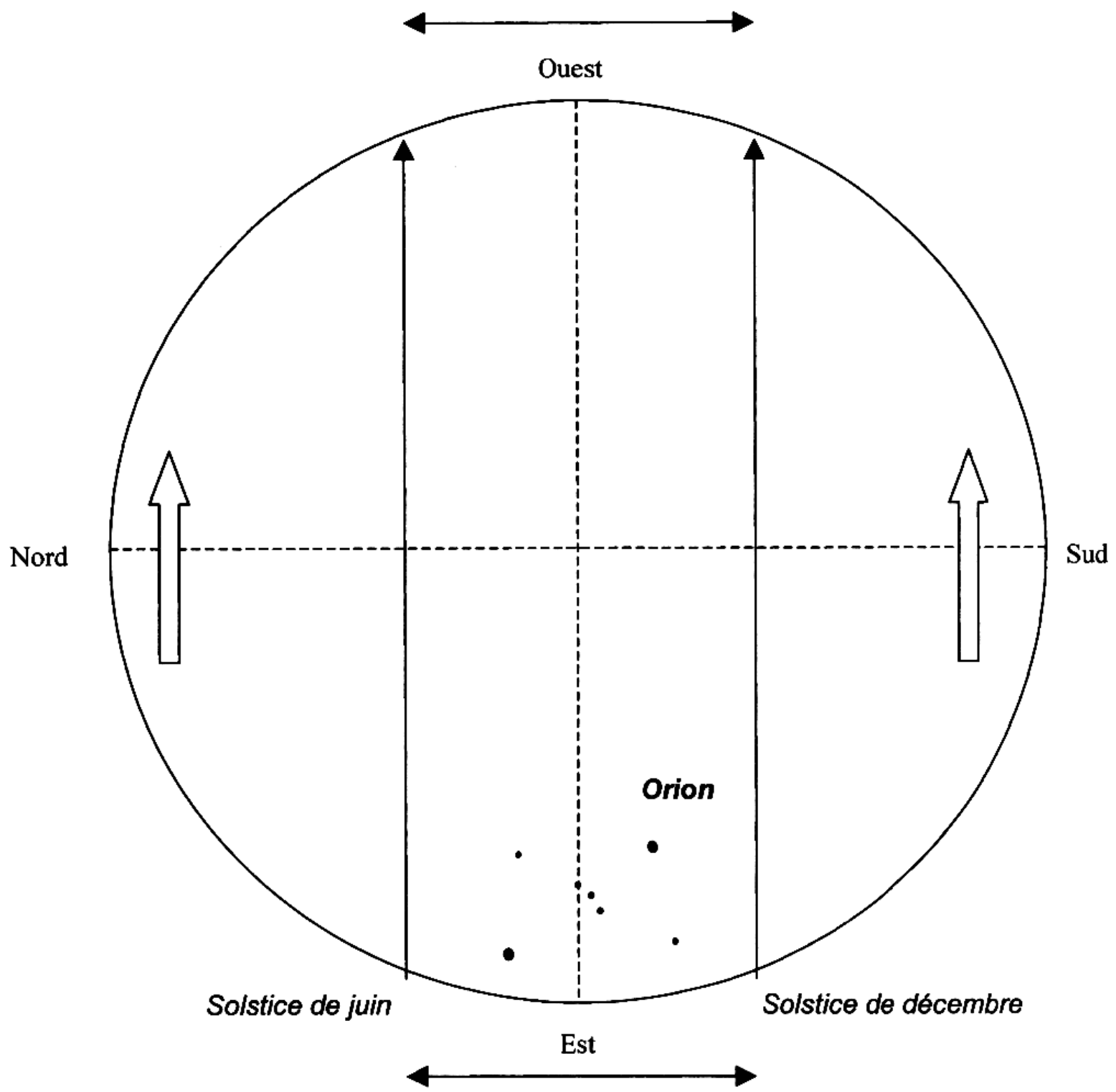

FIG. 8. - Orion dans une vue générale du ciel étoilé (élévation 90\%) autour de l'équateur. L'axe est-ouest divise le ciel en deux parties égales, et forme l'équateur céleste. Les directions nord et sud sont des points fixes autour desquels se " déroulent » les étoiles (d'est en ouest).

Le cercle extérieur représente la ligne d'horizon.

Entre le début et la fin de la nuit, la disposition d'une constellation se trouvera renversée suivant son point d'apparition et de disparition ${ }^{16}$.

\section{Structure de la constellation d'Orion}

Orion possède une disposition particulière autour du parcours équinoxial $\mathrm{du}$ soleil, c'est-à-dire une symétrie par paire d'étoiles de part et d'autre de l'axe est-ouest des quatre étoiles principales de la constellation. De plus, les points d'horizon marquant l'axe équinoxial représentent également, de nuit, l'équateur céleste. 


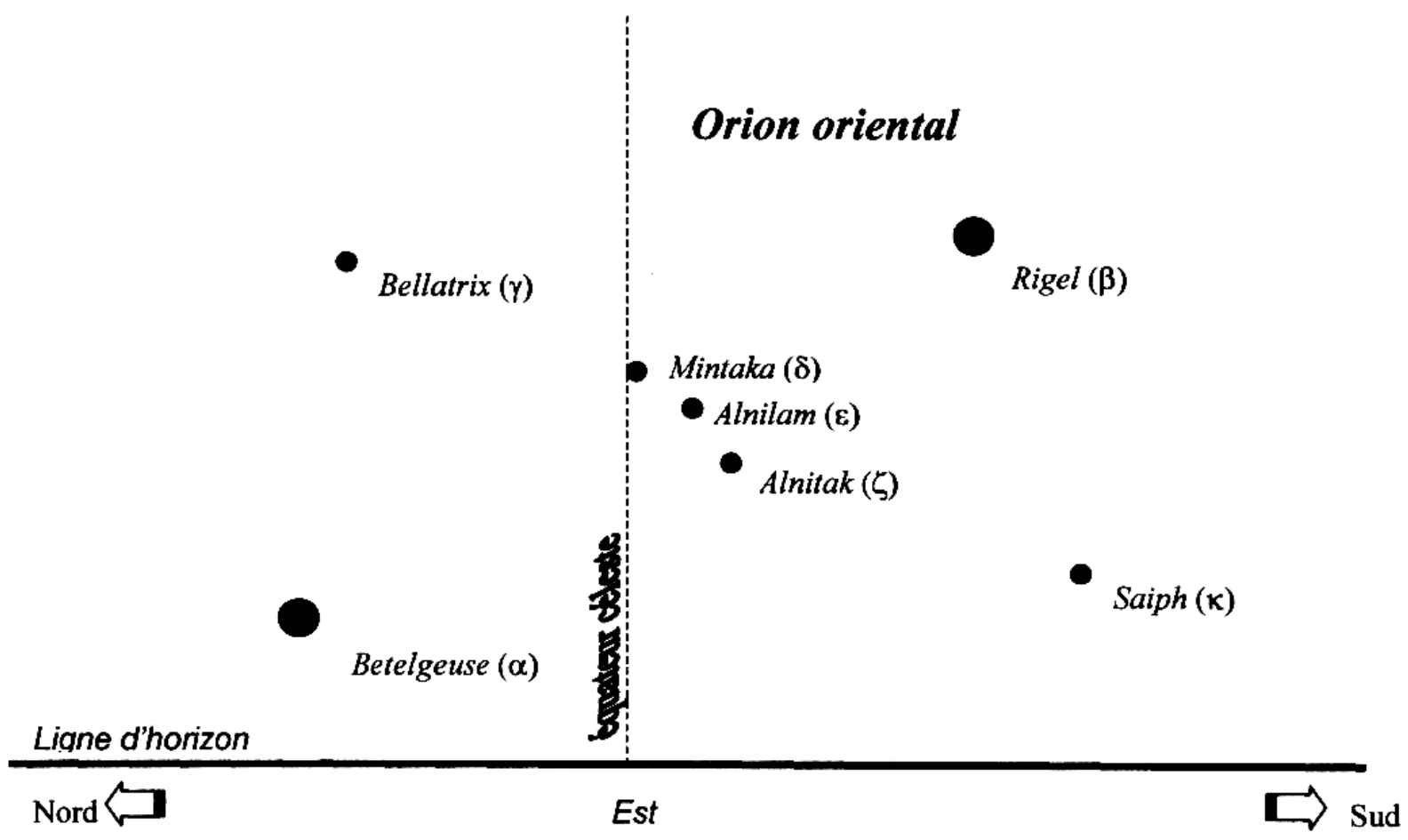

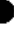

Saiph (к)
- Alnitakk $(\zeta)$

- Alinilam $(\varepsilon)$

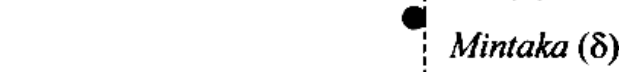

C

Rigel ( $\beta)$
Orion occidental

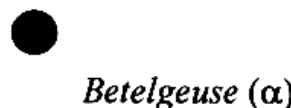

Betelgeuse $(\alpha)$

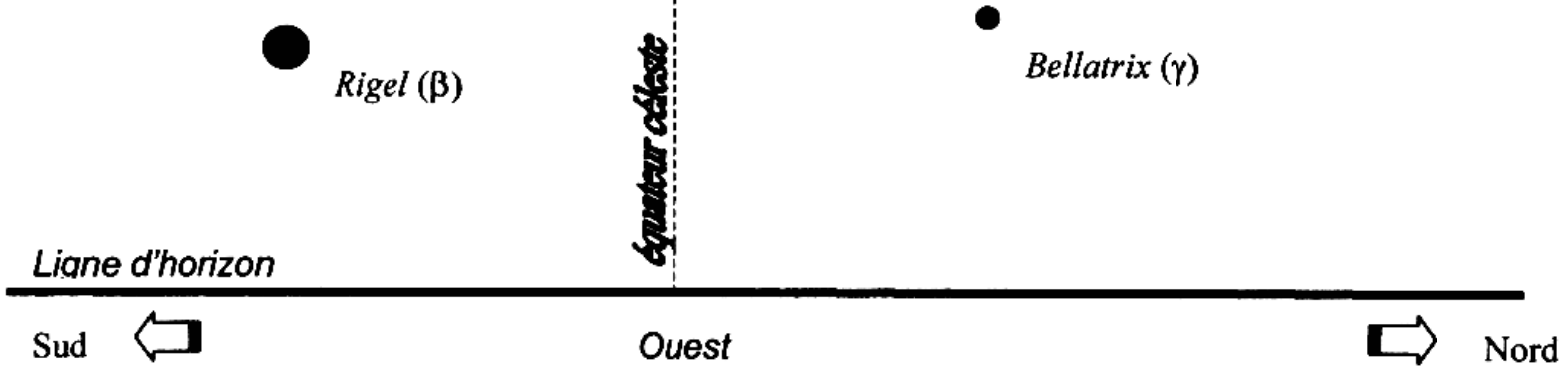

Fig. 9. - Orion - sans tracé occidental - à l'Est (trapèze à la base la plus courte vers le haut) et à l'Ouest (base la plus courte du trapèze vers le bas). 
Orion forme donc deux trapèzes suivant sa position dans le ciel (la petite base du trapèze vers le haut lorsque la constellation est " montante », à l'Est ; la petite base vers le bas lorsque la constellation est descendante, " tombante », à l'Ouest).

L'association de la constellation avec les points cardinaux est-ouest est forte, car l'apparition et la disparition équinoxiale du soleil se font exactement, sur la ligne d'horizon, à un point qui se trouve au centre de la constellation (bien que l'apparition et la disparition de la constellation et des deux astres majeurs - soleil et lune soient espacées de quelques heures). Si l'apparition et la disparition du soleil se font, au cours d'une année, entre les deux points extrêmes sur la ligne d'horizon marquant les deux solstices, l'apparition de la constellation d'Orion est, elle, invariable dans sa polarité, même si elle varie dans sa temporalité. Le centre de la constellation d'Orion coïncide constamment avec l'axe équinoxial ${ }^{17}$ (voir également Magaña, 1988: 80 et passim).

\section{Cyclicité de la constellation d'Orion : ascendante — descendante sur une année}

La présence dans le ciel étoilé de la constellation d'Orion associée au soleil divise l'année en deux périodes qui se trouvent en opposition complémentaire. La première va de la troisième semaine de juin à la troisième semaine de décembre ; c'est alors que la constellation apparaît dans le ciel à l'Est avant le soleil. Durant cette période, la constellation - lors de son lever héliaque - précède le soleil et semble le " porter " ou le " tirer» de dessous la terre pour le mettre dans le ciel. Ce phénomène a lieu avec quelques dizaines de minutes d'écart lorsque la constellation précède le soleil, mais peut également s'effectuer avec plusieurs heures de décalage, alors que la constellation se trouve déjà dans sa phase descendante à la fin de la nuit (elle se trouve alors à l'ouest du ciel étoilé). La caractéristique la plus importante de cette moitié de l'année est que la constellation d'Orion ne disparaît pas du ciel à l'Ouest avant le lever du soleil.

La seconde période couvre l'autre moitié de l'année ${ }^{18}$, durant laquelle la constellation d'Orion disparaît du ciel étoilé - par l'Ouest - avant que le soleil n'apparaisse. Durant cette période la constellation apparaît déjà dans le ciel lorsque le soleil se couche, et est donc dissociée de l'astre diurne. À l'aube, la constellation a déjà disparu du ciel ; elle ne peut donc plus être associée à l'astre diurne.

Les deux périodes peuvent ainsi être caractérisées suivant la polarité avec laquelle la constellation est associée : apparition à l'Est pour la première période (dans ce cas elle peut être désignée comme " montante » et associée au soleil, la base la plus courte du trapèze vers le " haut ») et disparition à l'Ouest pour la seconde (elle peut alors être qualifiée de « descendante » et dissociée du soleil, la base la plus courte du trapèze vers le «bas »).

Or nous avons vu avec le cas miraña que, lorsque la constellation est dans sa phase descendante (disparition occidentale), elle est associée à l'astre lunaire qui, comme le reste des corps célestes nocturnes, suit un parcours sidéral d'est en ouest.

Dans la mesure où il existe une opposition complémentaire entre soleil et lune (opposition nocturne / diurne), il semble que la seconde phase de la constellation d'Orion - sa phase descendante - ne soit pas liée à sa dissociation de l'astre solaire, mais bien plus à son association avec l'astre lunaire. En d'autres termes, durant la seconde phase, Orion ne précède pas le soleil, puisqu'il disparaît du ciel avant le lever 
du soleil, mais précède la lune ou, comme l'indique le mythe miraña : Astre (lune) est à la « poursuite » des quatre singes (Orion).

Il deviendrait alors possible pour la pensée mythique de raisonner en termes oppositionnels : ce que la constellation d'Orion réalise à l'Ouest avec la lune durant une moitié de l'année est l'équivalent de ce qu'elle réalise à l'Est avec le soleil pour la seconde moitié. Exprimé différemment, si les quatre singes tirent la tête de Lune à l'Ouest vers l'inframonde, ils font de même avec l'astre solaire mais en le faisant cette fois-ci sortir de cet inframonde ${ }^{19}$.

Les dispositions du trapèze d'Orion sont ainsi inversées suivant leur polarité et associées à l'un des deux astres majeurs :

a) Est, trapèze d'Orion " ascendant ", Soleil (premier semestre, troisième semaine de juin à troisième semaine de décembre).

b) Ouest, trapèze d'Orion « déclinant ", Lune (second semestre).

Suivant ces deux phases, et dans la mesure où, dans les pièces archéologiques que nous venons d'étudier, Orion est représenté par des singes en position tête-bêche, cette disposition pourrait indiquer la phase ascendante et déclinante de la constellation au long d'un cycle annuel.

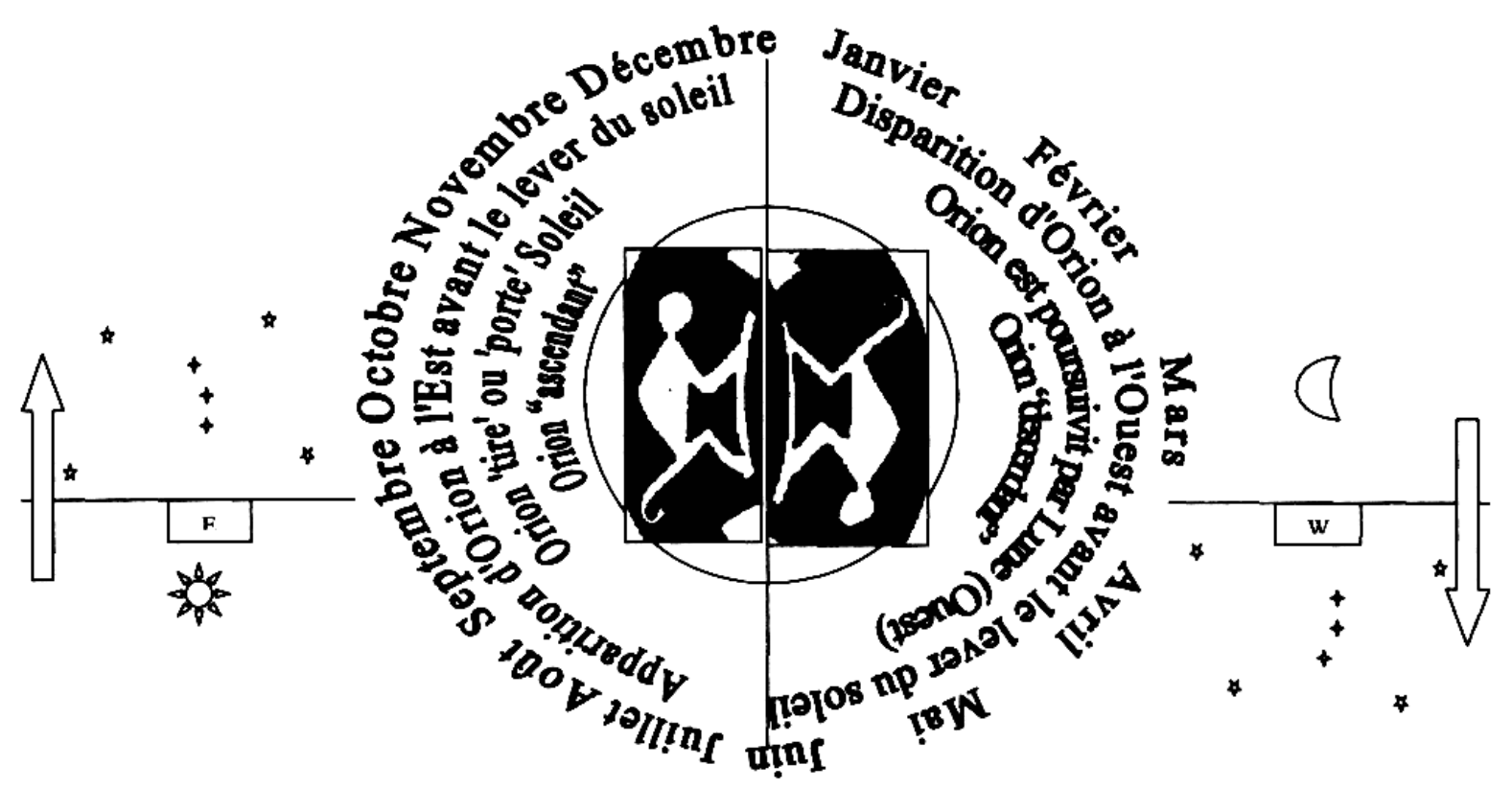

FIG. 10. - Cycle annuel d'Orion associé aux deux astres majeurs.

Il résulte finalement des constatations astronomiques que la constellation d'Orion est associée aussi bien à la lune qu'au soleil, et que cette association peut s'exprimer à l'aide du même motif mythique, à savoir la décapitation, la strangulation ou le transport d'un personnage astral (figurant aussi bien sa valeur diurne que nocturne) par quatre personnages situés symétriquement par paire de chaque côté de ce premier et dotés, le plus souvent, d'un comportement belliqueux à son encontre. Il existe un moyen encore plus simple de figurer cette association entre la constellation et les deux 
astres majeurs en disposant quatre étoiles - où tout ce qui peut en revêtir la valeur en trapèze autour d'un personnage central, voire même sur celui-ci.

Dans ce cas de figure, je tenterai de montrer dans un prochain travail que c'est l'ensemble des pièces archéologiques en provenance du continent sud-américain situées de part et d'autre de l'équateur (15 degrés nord et sud, après quoi la constellation change de disposition) et qui possèdent peu ou prou cette configuration qu'il conviendrait peut-être d'interpréter dans ce sens.

En nous cantonnant ici à la seule région archéologique proche de l'équateur, comme c'est le cas pour la région du Carchi-Nariño, la cyclicité de la constellation d'Orion est loin d'être anodine pour le découpage de l'année qu'elle permet. En effet, les dates du 21 juin et 22 décembre sont également les moments solsticiaux, c'est-àdire que le soleil atteint ses points les plus extrêmes d'apparition et de disparition vers le Sud et vers le Nord sur la ligne d'horizon. En d'autres termes, il est possible d'associer les phases d'Orion avec les deux solstices. Ainsi, pour les Desana du Vaupès - groupe de langue tukano situé également sur l'équateur -, Reichel-Dolmatoff évoque une association similaire entre cette constellation et les deux solstices : « [...] Betelgeuse et Bellatrix sont les points du solstice d'été, Saiph et Rigel les points du solstice d'hiver et la ceinture d'Orion est appelée "Chemin du Soleil ». Au centre [de la maloca], en Epsilon d'Orion, à certaines occasions rituelles - aux deux dates de l'équinoxe - , des tas de fruits de palmier [parépou] sont déposés. " (notre traduction, 1982 ;173). Or nous avons vu avec le mythe miraña que cette association entre les fruits de palmier et la constellation d'Orion passe par une décapitation du personnage Astre, c'est-à-dire lorsqu'il se trouve au centre de la constellation aux moments des équinoxes. Les dates des équinoxes coïncident par ailleurs avec les périodes de maturation des palmiers bactris (mars et septembre) permettant de voir dans cette conjonction une évocation du code temporel : le racème devient ainsi équivalent à la tête d'Astre. Suivant la disposition de la constellation de part et d'autre de l'équateur céleste, Epsilon d'Orion marque constamment, à un degré et demi près, les points de sorties équinoxiales ; ce qui laisserait penser que les Desana (par ailleurs voisins des Miraña) font la même association entre Orion, fruits de palmier et Astre, et ce, plus particulièrement lors des solstices et équinoxes.

Si nous insistons sur ce point, c'est que les motifs des poteries des figures 4 et 11 semblent indiquer cette association.

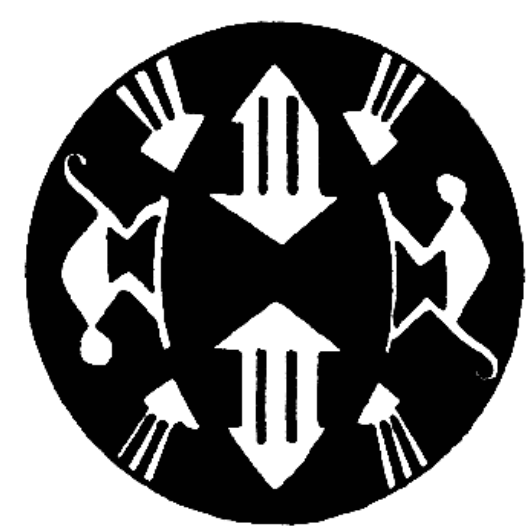

FIG. 11. - Intérieur de coupe Piartal ; deux singes têtes-bêches, $\mathbf{4}$ astres tronqués. 
Commençons par le motif de la figure 11. Si nous partons du fait que les représentations des singes à l'intérieur des poteries se réfèrent à Orion, il nous faut interpréter les représentations des quatre quarts de cercle dont émanent des rayons vers l'extérieur du contenant. Il semble qu'il s'agisse là de soleils partiellement tronqués, comme s'ils devaient représenter leurs levers et couchers derrière la ligne d'horizon ; les représenter, donc, sous forme de demi-cercles, et même, puisqu'il s'agit d'un pays montagneux où la ligne d'horizon n'est pas plane ${ }^{20}$, sous forme de quarts de cercles imparfaits et irradiants. Ces soleils sont répartis de part et d'autre de diagonales divisant la poterie. Si l'on suit le trajet du soleil durant les deux solstices, les quatre points d'intersections qu'ils forment sur la ligne d'horizon se répondent diamétralement d'un solstice à l'autre (le point d'horizon marquant le lever lors du solstice de décembre est diamétralement opposé au point d'horizon marquant le coucher lors du solstice de juin). En supposant que la poterie représente une vision globale du ciel, cette perception des quatre "soleils solsticiaux " serait parfaitement rendue par ce motif. Il est donc fort probable que la poterie (Figure 11) représente l'association entre les phases ascendante et descendante d'Orion (les deux représentations de singes tête-bêche, situés chacun sur un arc de cercle reliant chacun des soleils - parcours du soleil sur un solstice -) avec les deux périodes solsticiales (les quatre soleils) ; les équinoxes sont marqués par les pointes des triangles parvenant jusqu'à son bord (équidistants entre deux soleils solsticiaux) et représentant certainement le sommet d'une montagne (point équinoxial sur l'horizon montagneux).

Il faudrait cependant que l'ensemble de la zone couverte par le parcours du soleil sur la ligne d'horizon puisse être clairement représenté sur un motif de l'intérieur d'une coupe de cette région pour que l'hypothèse de cette association entre les deux phases d'Orion et les périodes solsticiales soit validée. L'intérieur de la poterie Piartal que nous avons présenté plus haut (Figure 4) semble remplir ces caractéristiques. Nous admettrons que, dans un rapport de métonymie, la chauve-souris est une évocation du ciel nocturne, et que le motif de la poterie (Figure 4) représente, comme nous l'avons suggéré, une combinaison de deux singes dont les membres servent également de membres au chiroptère. Les deux bandes blanches, parsemées de rectangles noirs alignés partant de chaque extrémité du corps du chiroptère et s'évasant lorsqu'elles rejoignent les bords du contenant, indiquent clairement une telle zone surtout lorsqu'elles sont associées au cercle blanc du centre de la poterie. De plus, l'alignement des rectangles noirs semble suivre un tracé que réaliserait un des deux astres majeurs dans le ciel (il faut noter que la lune évolue également dans le ciel nocturne au sein de cette zone marquée, dans le ciel diurne, par les deux trajets solsticiaux). C'est donc l'ensemble de cette zone qui serait ici soulignée, puisque les deux astres majeurs y évoluent, tout comme l'ensemble des constellations liées à l'équateur céleste, dont Orion, les pléiades et la planète Vénus. Dans ce cas, il est possible de donner une orientation aux faces internes de poteries en provenance de cette région ; la division diamétrale ne représente pas une vision " en coupe " d'une terre horizontale avec chacun des singes évoluant en direction inverse sur, et en dessous de la terre (hypothèse défendue par Uribe Alarcon dans son analyse de l'iconographie des poteries du Nariño ; 1988), mais d'une représentation du ciel nocturne.

On pourrait objecter à notre interprétation d'être par trop naïve et de prendre les représentations de l'intérieur des poteries pour ce qu'elles sont suivant des analogies 
" évidentes ", à savoir le fond noir pour le ciel nocturne, les zones en négatif comme devant représenter les parties brillantes de ce ciel, le cercle central comme étant un des deux astres majeurs, etc. Il faut cependant admettre que si les pendentifs dorés possèdent une quelconque relation avec ces intérieurs de poteries, ce qui semble être le cas puisqu'ils utilisent au moins le thème des singes, ces derniers y sont toujours représentés dans un déplacement vertical et non horizontal étant donné que ces ornements étaient suspendus, qu'il est donc possible de leur donner une orientation. Les poteries devraient donc être regardées en plaçant la bande centrale verticalement et, dans ce cas, cette bande ne peut plus représenter une coupe du disque terrestre. Le déplacement de singes sur un tronc rectiligne est ainsi plus probable, mais il faut alors prendre en compte les étoiles figurées aux alentours des singes, ou au centre de la bande. Quoi qu'il en soit, nous retournons alors à une perception similaire à celle exprimée dans le mythe miraña.

\section{CONCLUSION}

L'interprétation générale de ces pièces ornementales doit donc prendre en compte l'association première entre la constellation d'Orion avec les deux astres majeurs. Le fait que ces ornements de visages aient été retrouvés dans des tombes laissent supposer qu'ils pourraient avoir eu une utilité dans le transport de l'âme, au-delà de leur utilisation du vivant de leur possesseur. Là encore, c'est un parallèle avec les conceptions eschatologiques miraña qui nous autorise à faire ce rapprochement. Avant que l'influence croissante des croyances chrétiennes ne vienne bousculer une partie des croyances miraña, le chef de maloca était enterré au centre de celle-ci - conçue, par métaphore, comme son "monde »-, à la croisée des quatre poteaux centraux. Toujours selon les dires des intéressés, le parcours de l'âme de ce représentant du soleil sur terre était pris en charge par la constellation des Quatre Singes, située de part et d'autre des « portes » d'entrée et de sortie du ciel (cette partie du ciel comprise entre les points d'horizon des deux solstices). Son âme venait s'ajouter sous forme d'étoile à celles déjà existantes depuis l'origine des temps où le premier ordonnancement cosmique a donné naissance aux constellations. En ce sens, les ornements faciaux en provenance de cette région des Andes colombiennes pourraient signifier l'identification astrale de leur possesseur tout en servant de psychopompe au moment du trépas.

Les propositions ici exprimées pourraient avoir des implications majeures pour l'archéologie et l'ethnographie d'un grand ensemble géographique. Les rapports qu'entretenaient les deux régions principales dont il est formé (hautes terres/basses terres) n'étaient pas uniquement commerciaux mais auraient inclus également des conceptions symbolico-religieuses. La présence plutôt surprenante dans l'iconographie des hautes terres colombiennes d'animaux des basses terres pourrait ainsi s'expliquer par l'existence d'un ensemble mythologique et eschatologique ayant circulé le long des axes fluviaux pour parvenir jusqu'aux Andes ; on pourrait aussi supposer que ces conceptions auraient d'autant mieux été acceptées qu'elles se seraient greffées sur d'autres, déjà présentes et plus ou moins voisines.

L'interprétation iconographique des pièces archéologiques - qu'il s'agisse d'ornements en or ou de motifs à l'intérieur de poteries - ne doit donc pas faire 
l'économie d'une perspective astronomique. Dans l'hypothèse que nous défendons, il s'agit de véritables « astroglyphes " à comprendre comme des icônes et non comme des symboles puisqu'ils donnent à voir et entretiennent un rapport de ressemblance avec la réalité évoquée à travers leur structure. *

* Manuscrit reçu en juin 1999, accepté pour publication en juin 1999.

\section{NOTES}

1. Les premières idées de cet article ont été exposées en avril 1998 à la Société des Américanistes. Je tiens à remercier Jean-Pierre Chaumeil, Philippe Erikson et France-Marie Renard-Casevitz pour les remarques et commentaires qu'ils ont bien voulu me faire lors des différentes étapes d'écriture de ce texte, ainsi que Roberto Pineda et Maria Alicia Uribe pour m'avoir permis d'examiner les pièces du musée de l'Or de Bogota.

2. Une des dernières en date, et non des moindres, est l'analyse proposée par G. Reichel-Dolmatoff dans Orfebrería y chamanismo (1990) qui prend comme point de référence interprétatif les systèmes symbolicoreligieux - et plus particulièrement le chamanisme, comme le titre de l'ouvrage le laisse entendre - des sociétés indigènes actuelles de Colombie (en l'occurrence, les Kogi de la Sierra Nevada de Santa Marta, qui occupent aujourd'hui une partie du territoire de l'ancienne culture tairona, ainsi que les Desana d'Amazonie colombienne, ces deux sociétés ayant constitué ses principaux terrains d'enquêtes).

3. Une analyse détaillée de la répartition des espèces représentées dans l'art nariño indique que près des deux tiers de celles-ci possèdent une origine non andine (Rodríguez Bastidas, 1992).

4. "Las fuentes documentales sugieren también la existencia de una activa red de intercambio de los grupos étnicos del Putumayo y Caquetá con los pobladores de la juridicción de Pasto, que debió surgir de épocas prehispanicas si se observa, por ejemplo, la conocida tradición cerámica de "Los Pastos », caracterizada, entre otros aspectos, por su pintura al negativo. Esta cerámica, con detalles de fino trazo de las líneas, se lograba por el sistema de pintura al negativo, para lo cual necesariamente se empleaba cera de abejas que tradicionalmente se obtenía por intercambio con los grupos indigenas del río Caquetá y de sus afluentes. " (Gómez, $1996: 66$ )

5. Ce qui nous fait pencher vers cette interprétation, plutôt que pour un épi de maïs par exemple, est le fait que cette figuration reprenne les traits caractéristiques des racèmes de palmiers: succession de fruits globuleux sur le rachis du racème et, surtout, des ajours apparaissant clairement entre ceux-ci. L'identification avec Bactris gasipaes est une extrapolation de notre part à partir du mythe miraña ; n'importe quel autre racème de palmier à gros fruits globuleux ferait l'affaire (Mauritia flexuosa, par exemple).

6. Sur certaines de ces boucles d'oreilles (lc) les singes ont été remplacés par des figurations de volatiles alors que le même motif central a été gardé. Nous montrerons dans un autre travail que cette substitution n'est pas incompatible avec l'interprétation générale proposée, dans la mesure où d'autres sociétés précolombiennes, comme celle productrice de l'art Cauca, ont créé sur des pièces d'orfèvreries des êtres hybrides corps de singe / tête de volatile pour occuper et signifier la même association entre une espèce particulière de singe et un oiseau solaire. Il existe également des ornements d'oreilles dépourvus de singes ou de volatiles provenant de la même zone et de la même période reprenant cependant le personnage central à tête enflée placé en dessous de l'arc de cercle (Verneau \& Rivet, 1912 : planche XXIV, fig. 6), d'autres dont le racème de palmier est réduit à quatre fruits avec la base du rachis (ibidem, fig. 8) et d'autres, enfin (ibidem, fig. 7), qui répètent la forme du pendentif $1 \mathrm{~g}$, mais dont la tête est figurée par deux demi-sphères en relief en lieu et place de la simple tête en relief.

7. Une version plus complète du mythe, avec ses variantes, se trouve dans Karadimas 1997.

8. La confusion entre les deux espèces est d'ailleurs commune dans le massif colombien puisqu'elles portent souvent le même nom de " singe nocturne "; les miraña classent Poto flavus parmi les singes, ce qui est rendu dans le mythe par la relation de germains (classificatoires) instituée entre les deux espèces. Le kinkajou ne fait pas partie des primates mais possède les membres antérieurs en forme de mains ; de plus, et de par son comportement nocturne, ses yeux sont également surdéveloppés, à l'identique des douroucoulis. La possession d'une queue préhensile absente chez Aotus sp. marque une différence notoire. Il semble d'ailleurs que le pendentif d'oreille le ait figuré le kinkajou plutôt que le douroucouli : l'animal possédant un museau prononcé alors que les singes en sont exempts. 
9. " $\mathrm{El}$ " mono de noche " o « tutamono », Aotus trivirgatus (Cebidae) es una de las pocas especies de primates por cuya distribución es posible encontrarlo incluso en altitudes de hasta $3200 \mathrm{msnm}$ (...).El colgante orfebre Capuli 84 [du même type que 1f] muestra una pareja de monos con cabeza redonda, enormes ojos globulosos - adaptados para ver en la obscuridad - y cola grande no prensil, cualidades morfológicas típicas de esta especie. (Rodríguez B., 1992 : 75) ").

10. On pourrait aussi être tenté d'associer à cette dernière représentation l'intérieur d'une autre poterie Piartal - non illustrée ici - au motif identique de singes tête-bêche, de part et d'autre d'une bande centrale, où l'on observe trois cercles alignés mais perpendiculairement à la bande et qui pourraient représenter le Baudrier d'Orion (in Bray, 1998 ; Fig. 4c).

11. Nous montrerons dans un autre travail comment cette représentation combinée des deux groupements d'étoiles existe pour les populations d'autres régions des Andes colombiennes. Il existe également provenant des Andes équatoriennes une représentation de l'ensemble de la constellation sous la forme d'une hache cérémonielle dépourvue de fil, portée en pendentif, (aujourd'hui au Musée de l'Homme) qui possède, selon une description de P. Rivet, « (des ornements à jour) constitués par trois petits trous circulaires, placés en ligne verticale suivant l'axe du talon, et par quatre orifices cruciformes » (pl. XXV, fig. 8) qui forment, peut-on ajouter, un trapèze encadrant les trois trous alignés verticalement à la façon des étoiles de la constellation (1912: 272). Les autres motifs existant sur des haches cérémonielles de la même région ne seraient que des variations plus complexes de ce motif de base. On peut noter que de cette même région des Andes équatoriennes proviennent deux pectoraux discoïdes représentant chacun quatre félins disposés symétriquement à la manière des singes du Nariño dont les têtes forment un trapèze sur chacun des disques (ibidem : pl. XXIII, fig. 1, pl. XXIV, fig. 3).

12. Pour expliquer cet effet secondaire de la bière de parépou, les Miraña évoquent le mythe. Le fils d'Astre récupérera du monde des poissons un noyau de bactris (brûlant car solaire) en l'avalant pour le soustraire aux yeux des poissons - ses alliés - . Saisi de violentes diarrhées une fois revenu sur terre, il déféquera le noyau du premier bactris terrestre.

13. Pour plus de détails sur ces associations entre cervelle et noyaux, voir Karadimas 1997. On retiendra cependant que cette association s'appuie sur un lien établi par les Miraña entre le sperme que la cervelle est censée produire en partie et les noyaux considérés comme des semences dans les deux sens du terme.

14. Des pendentifs d'oreilles en provenance de la province du Nariño ( $n^{\circ} 25393$ \& -94 du Musée de l'Or de Bogotá) et remontant également à la période Capulí représentent une tête en relief entourée d'une multitude de visages avec une orientation concentrique dans une configuration similaire à celle des soleils de la figure ld, sur laquelle le soleil en relief est entouré de rayons dont les extrémités sont rehaussées de cercles également en relief.

15. Dans le cas miraña qui nous préoccupe, on citera les formations rocheuses d'Araracuara. À l'inverse, et dans un schéma plus classique, les étoiles servent d'éléments d'orientation pour les sociétés de forêt chez lesquelles les déplacements des sites d'habitations sont plus fréquents que les villages stables et pour lesquelles la ligne d'horizon est absente ou toujours couverte par la cime des arbres.

16. Il faut noter que la disposition des étoiles - hormis les planètes donc - ne varie que très faiblement avec le cours des siècles. Ainsi, le ciel étoilé vu par les sociétés amérindiennes y a deux mille ans est similaire à celui d'aujourd'hui ; nous donnons ici une représentation du ciel autour de l'an 1000 .

17. Il existe, comme nous pouvons le remarquer sur les images astronomiques, une différence de $1^{\circ} 30^{\prime}$ entre l'Est réel et le centre d'Orion, sur laquelle nous reviendrons.

18. Sauf entre mi-mai et mi-juin, lever cosmique pendant lequel le soleil et Orion sont trop proches au lever du jour.

19. Dans ce cas Orion permet le lever du soleil; naturellement, il resterait à savoir qui permet ce lever dans la seconde partie de l'année pendant laquelle Orion est associé à l'astre nocturne.

20. Plusieurs volcans éteints possèdent entre El Carchi et le Nariño des pics de près de $4800 \mathrm{~m}$.

\section{BIBLIOGRAPHIE}

ANDRADE, Angela, 1986. - Investigación Arqueologica de los Antrosoles de Araracuara, Fundación de Investigación Arqueológicas Nacionales, Banco de la República, Bogotá. 
ARIAS DE GRIEFF, Jorge \& Elizabeth ReICHEL (Compiladores), 1987. - Etnoastronomias americanas, Ed. Universidad Nacional de Colombia, Bogotá.

AVENI, Anthony F. \& Gary URTON (Editors), 1982. - Ethnoastronomy and Archeoastronomy in the American Tropics, Annals of the, vol. 385, New York Academy of Sciences, New York.

Bray, Tamara L., 1998. — «Monos, Monstruos, y Mitos : Conexiones Ideológicas Entre la Sierra Septentrional y el Oriente del Ecuador ", in : CÁrdenAS-Arroyo \& Bray (Ed.), Intercambio y Comercio entre Costa, Andes y Selva. Arqueologia y Etnohistoria de Suramérica, Dpt. de Antropología, Universidad de los Andes, Bogotá, pp. 135-154.

Cárdenas-Arroyo, Felipe \& Tamara L. Bray (Ed.), 1998. - Intercambio y Comercio entre Costa, Andes y Selva. Arqueología y Etnohistoria de Suramérica, Dpt. de Antropología, Universidad de los Andes, Bogotá.

Emmons, Louise H., 1990. - Neotropical Rainforest Mammals. A Field Guide, The University of Chicago Press Ltd., London.

GASCHÉ, Jürg et al. (ETSA), 1996. — « Los alcances de la noción de « cultura » en la educación intercultural. Exploración de un ejemplo : sociedad y cultura bora ", in : GodENZzi, A. (Complilador), Educacion e interculturalidad en los Andes y la Amazonia, Estudios y debates regionales Andinos, $\mathrm{n}^{\circ}$ 93, Centro de Estudios Regionales Andinos Bartolomé de Las Casas, Cusco, Pérou.

Gómez LoPEZ, Augusto J., 1996. — « Bienes, rutas y mercados (siglos XV-XIX). Las relaciones de intercambio interétnico entre las tierras bajas de la Amazonía y las tierras altas de los Andes ", Revista de Antropologia y Arqueología, vol. IX, ${ }^{\circ s}$ 1-2, Universidad de los Andes, Bogotá, pp. 51-80.

Guyor, Mireille, 1974. - «La maison des indiens Bora et Miraña », Journal de la Société des Américanistes, vol. 61, pp. 141-176.

—, 1976. - « Structure et évolution chez les Indiens Bora et Miraña. Amazonie colombienne », Actes du XLII Congrès International des Américanistes, II, Société des Américanistes, Paris, pp. $163-173$.

HILDEBRAND, Martin von., 1987. - « Datos etnograficos sobre la astronomía de los indigenas Tanimuka del Noroeste Amazonico ", in : ARIAS DE GRIEFF \& ReICHEL (Compiladores), Etnoastronomías americanas, Ed. Universidad Nacional de Colombia, Bogotá, pp. 233-254.

KaradimaS, Dimitri, 1997. - Le Corps Sauvage. Idéologie du corps et représentations de l'environnement chez les Miraña d'Amazonie colombienne, Thèse de doctorat nouveau régime, Université Paris X-Nanterre, 2 vol.

Lathrap, Donald, 1970. - The Upper Amazon, Thames and Hudson, London.

Lavallée, Danielle \& Luis Guillermo Lumbreras, 1985. - Les Andes de la Préhistoire aux Incas, Gallimard, coll. « L'univers des formes ", Paris.

MaGaÑa, Edmundo, 1987. - "Astronomía y mitos Estelares de los Indios de las Guayanas ", in : ARIAS DE GRIEFF \& REICHEL (Compiladores), Etnoastronomías americanas, Ed. Universidad Nacional de Colombia, Bogotá, pp. 45-56.

—, 1988. - Orión y la mujer Pléyades. Simbolismo astronómico de los indios kaliña de Surinam, CEDLA, FORIS Publications $\mathrm{n}^{\circ} 44$, Dordrecht (Holland), Providence Rhode Island (USA).

MAGAÑA, Edmundo \& Peter Mason (eds.), 1986. - Myth and the Imaginary in the New World, CEDLA, FORIS Publications $\mathrm{n}^{\circ} 34$, Dordrecht (Holland), Providence Rhode Island (USA).

Meggers, Betty J., 1971. - Amazonia : Man and Culture in an Conterfeit Paradise, AldineAtherton, Chicago-New York. 
Ortiz, S. Elías, 1934. - «Los petroglifos de Negrohuaico ", Boletin de estudios históricos, Vol. V., n' 56 a 60 , pp. 313-317.

Porro, Antonio, 1984. - « Os Solimões ou Jurimaguas. Território, migrações e comércio intertribal ", Revista do Museu Paulista, 5 (56), pp. 23-38.

—, 1985. — «Mercadorias e rotas de comercio intertribal na Amazonia » Revista do Museu Paulista, vol. 30, pp. 7-12.

Ramírez de JARA, María Clemencia, 1996 a. - « Territorialidad y Dualidad en una Zona de Frontera del Piedemonte Oriental : El caso del Valle de Sibundoy ", in : CAIllavet \& PACHón (Comp), Frontera y Poblamento : Estudios de Historia y Antropología de Colombia y Ecuador, IFEA, SINCHI, Dpt. de Antropología, Universidad de los Andes, Bogotá, pp. 111-137.

—, 1996 b. - Frontera fluida entre Andes, Piedemonte y Selva : El Caso del Valle de Sibundoy, Siglo XVI-XVIII, Instituto Colombiano de Cultura Hispánica, coll. Cuadernos de Historia Colonial, Bogotá.

Reichel-DolmatofF, Gérard, 1982. - « Astronomical Models of Social Behavior Among Some Indians of Colombia ", in : AvENI \& URTON (Editors), Ethnoastronomy and Archeoastronomy in the American Tropics, Annals of the New York Academy of Sciences, vol. 385, New York, pp. 165-182.

—, I990. - Orfevrería y Chamanismo. Un estudio iconográfico del Museo del Oro, Banco de la Republica, Ed. Colina, Medellin, Colombia.

Renard-CaSEvitz, France-Marie, Anne-Christine Taylor \& Thierry SaIGNES, 1988. - Al Este de los Andes, IFEA-Abya-yala, Quito, Ecuador.

Rodriguez Bastidas, Edgar Emilio, 1992. - Fauna Precolombina de Nariño, Fundación de Investigaciones Arqueológicas Nacionales, Banco de la República, ICAN, Bogotá.

Uribe Alarcon, María Victoria, 1986. — « Pastos y Protopastos : la red regional de intercambio de productos y materias primas de los siglos X a XVI D.C. ", Manguaré, 3, Universidad Nacional de Colombia, Bogotá, pp. 33-46.

Uribe Alarcon, María Victoria \& Fabricio Cabrera Micolta, 1988. - «Estructuras de pensamiento en el altiplano nariñense : Evidencias de la arqueología ", Revista de Antropologia, vol. IV, $\mathrm{n}^{\circ} 2$, pp. 43-70.

Verneau, R. \& Rivet, Paul, 1912. - Ethnographie ancienne de l'Équateur, Gauthier-Villars, Paris.

Zuidema, Tom, 1989. - Reyes y Guerreros. Ensayos de cultura andina., Grandes Estudios Andinos, Fomciencias, Lima, Perú.

\section{CRÉDIT PHOTOGRAPHIQUE}

Les figures 1a, 1d, 1e, 1f, $1 \mathbf{g}$ et $\mathbf{1 h}-\mathbf{b}$ sont tirées de : L'or des Dieux, l'or des Andes. Pérou, Équateur, Colombie. Catalogue de l'exposition organisée par le conseil Général de la Moselle Metz-Arsenal, sous la direction de D. LÉviNE, I994, Ed. Serpentoise.

Les figures $1 \mathbf{b}$ et $1 \mathbf{c}$ sont tirées de : Civilisations du Soleil. Chefs-d'cuvre du Musée de l'Or de Bogotá. Catalogue de l'exposition organisée par la Ville de Toulouse et le Musée des Augustins, sous la direction de C. Plazas \& A. Daguerre de Hureaux, 1996, Ed. Adam Biro.

La figure 2a de Lavallée \& Lumbreras 1985 : 130, Photo 121. 
La figure 2b est tirée de : L'art précolombien, J. MazenOd \& J. Alcina, Mazenod Ed., 1978, Paris, Photo 607.

La figure 1h-a est tirée de Goldmuseum Kolumbien. Banco de la República, L. DuQue Gomez, Ed. Delroisse, 1982, Bogotá

Les figures 3a, 3b, 4, 7a et 11 sont tirées de : M.V. URIBE, 1988.

Les figures $\mathbf{1 i}, \mathbf{1} \mathbf{j}, \mathbf{5 a}, \mathbf{5 b}$ et $\mathbf{6}$ sont des reproductions à main levée, par l'auteur, des pièces du musée de l'Or de Bogota. 